\title{
Inferring the Inclination of a Black Hole Accretion Disk From Observations of Its Polarized Continuum Radiation
}

\section{Citation}

Li, Li-Xin, Ramesh Narayan, and Jeffrey E. McClintock. 2009. Inferring the Inclination of a Black Hole Accretion Disk From Observations of Its Polarized Continuum Radiation. The Astrophysical Journal 691, no. 1: 847-865. doi:10.1088/0004-637x/691/1/847.

\section{Published Version}

doi:10.1088/0004-637X/691/1/847

\section{Permanent link}

http://nrs.harvard.edu/urn-3:HUL.InstRepos:27814534

\section{Terms of Use}

This article was downloaded from Harvard University's DASH repository, and is made available under the terms and conditions applicable to Other Posted Material, as set forth at http:// nrs.harvard.edu/urn-3:HUL.InstRepos:dash.current.terms-of-use\#LAA

\section{Share Your Story}

The Harvard community has made this article openly available.

Please share how this access benefits you. Submit a story.

\section{Accessibility}




\title{
INFERRING THE INCLINATION OF A BLACK HOLE ACCRETION DISK FROM OBSERVATIONS OF ITS POLARIZED CONTINUUM RADIATION
}

\author{
Li-Xin Li ${ }^{1}$, Ramesh Narayan ${ }^{2}$, And JefFrey E. McClintock ${ }^{2}$ \\ ${ }^{1}$ Max-Planck-Institut für Astrophysik, Karl-Schwarzschild-Str. 1, 85741 Garching, Germany \\ 2 Harvard-Smithsonian Center for Astrophysics, 60 Garden Street, Cambridge, MA 02138, USA \\ Received 2008 September 4; accepted 2008 September 30; published 2009 January 19
}

\begin{abstract}
Spin parameters of stellar-mass black holes in X-ray binaries are currently being estimated by fitting the X-ray continuum spectra of their accretion disk emission. For this method, it is necessary to know the inclination of the X-ray-producing inner region of the disk. Since the inner disk is expected to be oriented perpendicular to the spin axis of the hole, the usual practice is to assume that the black hole spin is aligned with the orbital angular momentum vector of the binary, and to estimate the inclination of the latter from ellipsoidal modulations in the light curve of the secondary star. We show that the inclination of the disk can be inferred directly if we have both spectral and polarization information on the disk radiation. The predicted degree of polarization varies from $0 \%$ to $5 \%$ as the disk inclination changes from face-on to edge-on. With current X-ray polarimetric techniques the polarization degree of a typical bright X-ray binary could be measured to an accuracy of $0.1 \%$ by observing the source for about 10 days. Such a measurement would constrain the disk inclination to within a degree or two and would significantly improve the reliability of black hole spin estimates. In addition, it would provide new information on the tilt between the black hole spin axis and the orbital rotation axis of the binary, which would constrain any velocity kicks experienced by stellar-mass black holes during their formation.
\end{abstract}

Key words: accretion, accretion disks - black hole physics - polarization - radiative transfer - relativity - X-rays: binaries

\section{INTRODUCTION}

$\mathrm{X}$-ray polarimetry is an uncharted frontier with great promise for the exploration of the behavior of accreting black holes and for the measurement of black hole spin. To date, only small X-ray polarimeters have been flown and the only result of consequence has been the measurement of the polarization of the Crab Nebula (Novick et al. 1972; Weisskopf et al. 1978). Anticipating the launch of larger and more sensitive X-ray polarimeters in the future, this paper discusses possible uses of polarization observations to study thermal emission from accretion disks around stellar-mass black holes in X-ray binaries.

Polarization measurements at radio and optical wavelengths attest to the power of polarimetry. In radio astronomy, the measurement of polarization is trivial. However, its consequences have been of central importance since Alfvén \& Herlofson (1950) first suggested the synchrotron mechanism as the source of celestial radio emission. For example, studies of this strongly polarized radiation has allowed the mapping of magnetic fields that are threaded throughout stellar coronae, supernova shocks, relativistic jets, and the interstellar medium (ISM) and intergalactic medium (IGM). At optical wavelengths, the measurement of polarization is more difficult, but the payoffs have also been large. For example, polarimetric observations of NGC 1068 provided a spectacular confirmation of the unified model of active galactic nucleus (AGN; Antonucci \& Miller 1985). Polarization measurements are most challenging at X-ray wavelengths; however, sure instrumental approaches are known (Section 6), and the discovery space and potential rewards are large.

$\mathrm{X}$-ray polarimetric information-direction and degreeincreases the parameter space used to investigate compact objects from the current two-spectra and time variability- to four independent parameters that models must satisfy (Rees
1975; Lightman \& Shapiro 1975; Mészáros et al. 1988). $\mathrm{X}$-ray polarimetry can provide qualitatively new information on source geometry and magnetic fields on spatial scales comparable to a black hole event horizon. In this paper, we follow the pioneering work of Stark \& Connors (1977), Connors \& Stark (1977), and Connors et al. (1980), and we narrow our focus to consider solely the problem of polarized thermal emission generated by a thin accretion disk around a stellar-mass black hole. The theory developed here of course applies as well to thermal accretion disks around supermassive black holes. We do not consider coronal effects that can generate an X-ray reflection spectrum (e.g., Ross et al. 1999; Dovčiak et al. 2004) and Comptonized components of emission (e.g., Gierliński et al. 1999).

The polarization model presented here extends our workhorse accretion disk model KERRBB ( $\mathrm{Li}$ et al. 2005), which we have used to estimate the spins of four stellar-mass black holes via fits to their thermal continuum spectra (Shafee et al. 2006; McClintock et al. 2006; Liu et al. 2008). The extension of KERRBB presented herein includes a simple electron scattering atmosphere based on an $\alpha$-viscosity prescription of the stress (Shakura \& Sunyaev 1973) and a treatment of the polarized radiation field via the Stokes parameters. Radiative transfer effects will be considered in a future paper.

In Section 2, we discuss our motivations for this work, focusing in particular on the application of polarization measurements for estimating the spins of accreting black holes. In Section 3, we briefly review the polarization of disk emission induced by electron scattering in an accretion disk atmosphere, assuming a semi-infinite plane-parallel medium. In Section 4, we present the mathematical scheme for calculating the polarization as observed by a remote observer, after the polarized disk emission propagates through the curved spacetime of a Kerr black hole. In Section 5, we apply our polarization calculations to the measurement of black hole spin, and in Section 6, we briefly 
discuss the prospects for observing the predicted polarization signal from accreting stellar-mass black holes. Finally, in Section 7 , we summarize the results. Some mathematical details related to the thin disk model we employ and the propagation of the polarization vector in Kerr spacetime are presented in Appendices A and B.

\section{MOTIVATIONS}

There are two principal and related motivations for undertaking this work: (1) to validate a key assumption of the continuumfitting method of determining spin, and (2) to determine if the spins of stellar-mass black holes are aligned with the orbital plane. We discuss these topics in turn in the following subsections. We then conclude this section by commenting on three additional avenues for determining black hole spin, viz., the Fe $\mathrm{K}$ line, high-frequency quasi-periodic oscillations (QPOs), and $\mathrm{X}$-ray polarimetry.

\subsection{Validation of the Continuum-Fitting Method of Measuring Spin}

Using the continuum-fitting method, we have published spin estimates for four stellar black holes (Section 1), and we expect to publish spin estimates for several more during the next few years. In this work, it is essential to use thermaldominant (TD; formerly high soft state) data (McClintock \& Remillard 2006). A feature of this state is the dominance of a soft blackbody-like component that is emitted by optically thick gas in the accretion disk. A minor nonthermal tail component of emission is also often present, but it contributes typically only a few percent of the $\sim 1-10 \mathrm{keV}$ flux. Thus, these thermal-state spectra are largely free of the uncertain effects of Comptonization and are believed to match closely the classic thin accretion disk models of the early 1970s (Shakura \& Sunyaev 1973; Novikov \& Thorne 1973). There is an abundance of such suitable thermal-state continuum spectra available in the NASA HEASARC archives for most black hole binaries. These spectra are ideally suited for study using the polarization model presented in this paper.

The promise of the continuum-fitting method for determining black hole spin is attested to by 20 years of experience that has demonstrated for the TD state the presence of a stable inner disk radius (see Section 4 in McClintock et al. 2008). The inner edge is presumably closely tied to the innermost stable circular orbit (ISCO). X-ray observations, coupled with ground-based optical and infrared observations, are used to measure the radius of this orbit. Applying the continuum-fitting method is analogous to the familiar problem of determining the radius of a star of known distance given its effective temperature and flux (McClintock et al. 2008). Accordingly, this method requires knowledge of the luminosity of the X-ray source, which depends not only on its distance but also on the projected area and hence inclination of the accretion disk. Accurate values of the inclination of the orbital plane $i_{\text {orb }}$, distance $D$, and black hole mass $M$ are readily obtained from ground-based observations. This optical work has been underway for 35 years and is now undergoing a renaissance (e.g., Orosz et al. 2007).

The continuum-fitting method is straightforward to apply. All the required data are readily obtainable, and even the theory of disk accretion in strong gravity is tractable (Narayan et al. 2008; Shafee et al. 2008b). However, the reliability of the continuum-fitting method is called into question by a single assumption, viz., that the plane of the inner X-ray-emitting portion of the disk is aligned with the binary orbital plane, whose inclination angle $i_{\text {orb }}$ is determined from optical observations. Unfortunately, the continuum-fitting method cannot fit for the inclination of the inner disk and check for disk warp (Section 2.2) because there is a degeneracy between the inclination and spin parameters (Section 5.2; see also Liu et al. 2008). As we show, polarimetry observations can easily determine the inclination of the inner disk. This is our principal motivation for writing this paper.

\subsection{The Inclination of the Inner Accretion Disk}

Whether a black hole's spin is aligned with its accretion disk and the timescale for any misalignment to dissipate are topics that have received considerable attention recently (Fragile \& Anninos 2005; King et al. 2005; Lodato \& Pringle 2006; Fragile et al. 2007; Martin et al. 2007). In this paper, we restrict our attention to the question of alignment in the case of stellar black holes in X-ray binaries. If a black hole's spin was to be misaligned from the orbital vector, the inner disk would be warped away from the outer disk by the Lense-Thirring precession and the Bardeen-Petterson effect (Bardeen \& Petterson 1975), which would be expected to cause a global precession of the main body of the disk and have important observational consequences (e.g., enhanced mass accretion rate and jet precession; Fragile et al. 2007; Martin et al. 2007).

Are significant misalignments expected in the case of accreting stellar black holes? On the one hand, it seems plausible that the spin will be aligned with the orbit vector given the strong tidal forces acting on the pre-supernova star (Zahn 1977) and, as generally assumed (e.g., Fryer \& Kalogera 2001), that collapse of the stellar core to a black hole will be nearly spherical and mostly free of the strong shocks and velocity kicks that nascent neutron stars suffer. Even if shocks and kicks do occur, it is expected that their effects will be less for black holes by the factor $M_{\mathrm{BH}} / M_{\mathrm{NS}} \sim 7$ (e.g., Fryer \& Young 2007). On the other hand, there is some evidence for warped disks based on radio-jet data (Maccarone 2002), although this evidence is weak (Narayan \& McClintock 2005). One can attempt to infer inclination via studies of radio jets (Mirabel \& Rodríguez 1999) and $\mathrm{Fe} \mathrm{K}$ emission line profiles (Fabian et al. 2000). However, as we show in this paper, the direct and sure approach is by measuring the degree of polarization of the thermal accretiondisk radiation, which depends strongly on the inclination angle $i_{\text {disk }}$ of the inner disk.

\subsection{Three Additional Methods of Measuring Black Hole Spin}

The Fe $\mathrm{K}$ line method of determining spin is applicable to supermassive black holes as well as stellar black holes (Miller 2007; Reynolds \& Fabian 2008). It has received considerable attention and has recently yielded quantitative estimates of spin for the Seyfert 1.2 galaxy MCG-6-30-15 (Brenneman \& Reynolds 2006) and for the stellar black hole GX 339-4 (Miller et al. 2008).

High-frequency QPOs may develop into a precise method of determining spin once the correct model of the oscillations has been identified (e.g., Wagoner et al. 2001; Török et al. 2005; Schnittman 2005). X-ray polarimetry may also provide a means of determining spin. This possibility is implicit in the work of Connors et al. (1980), which shows that for reasonable assumptions one expects the plane of polarization to swing smoothly through a large range of angle as the photon energy increases. By modeling this effect and the degree of polarization one can hope to obtain 
an independent estimate of black hole spin, a topic that we touch upon in Section 5.1.

Thus, there are potentially a total of four methods of measuring black hole spin, two of which-the continuum-fitting method and the Fe K line method-are presently delivering results. Because black hole spin is such a fundamental parameter, it is important to attempt to measure it by as many of these methods as possible, as this will provide arguably the best possible check on the results.

\section{POLARIZATION OF DISK EMISSION INDUCED BY ELECTRON SCATTERING}

We discuss in this and the following section the technical details of how we calculate the polarization of the thermal disk emission. We present additional details in the Appendices. The presentation is pedagogical so that this paper and Li et al. (2005) together provide a complete and self-contained description of the calculations.

The radiation generated deep inside an accretion disk is initially unpolarized, but it becomes polarized as a result of electron scattering in the disk atmosphere. For an optically thick and geometrically thin accretion disk, the disk atmosphere is locally well described by a semi-infinite plane-parallel medium. We make this approximation.

A radiation field is completely described by four Stokes parameters: $I$ ( $\equiv I_{l}+I_{r}$, the total intensity), $Q$ ( $\left.\equiv I_{l}-I_{r}\right)$, $U$, and $V$ (Chandrasekhar 1960). Here, the subscripts $l$ and $r$ refer, respectively, to polarization in the meridian plane (i.e., the plane containing the symmetry axis and the line of sight to the observer) and right angles to it. In a plane-parallel atmosphere with no incident radiation, the axial symmetry of the radiation field requires that the plane of polarization be either along $l$ or $r$. Therefore, $U=V=0$, and the two parameters $I$ and $Q$, or equivalently the two intensities $I_{l}$ and $I_{r}$, suffice to characterize the radiation. The degree of polarization of the radiation field is then given by

$$
P \equiv \frac{1}{I}\left(Q^{2}+U^{2}+V^{2}\right)^{1 / 2}=\frac{\left|I_{r}-I_{l}\right|}{I_{r}+I_{l}} .
$$

At the surface of a pure scattering atmosphere, the two intensities $I_{l}$ and $I_{r}$ are determined by

$$
\begin{gathered}
I_{l}(\mu)=\frac{3}{8 \sqrt{2} \pi} F H_{l}(\mu) q, \\
I_{r}(\mu)=\frac{3}{8 \sqrt{2} \pi} F H_{r}(\mu)(\mu+c),
\end{gathered}
$$

where $\mu \equiv \cos \theta, \theta$ is the polar angle of the direction of propagation of the photon to the disk normal, $F$ is the total emitted flux density, $H_{l}$ and $H_{r}$ are Chandrasekhar's $H$ functions (Chandrasekhar 1960), and $q$ and $c$ are two integral constants. Following Chandrasekhar (1960), we have suppressed the dependences of the relations on the photon energy since no ambiguity is likely to arise. The constants $q$ and $c$ are given by (Chandrasekhar 1960, Section X)

$$
\begin{gathered}
q=\frac{8\left(A_{1}+2 \alpha_{1}\right)-6\left(A_{0} \alpha_{1}+\alpha_{0} A_{1}\right)}{3\left(A_{1}^{2}+2 \alpha_{1}^{2}\right)}, \\
c=\left(1-\frac{q^{2}}{2}\right)^{1 / 2}
\end{gathered}
$$

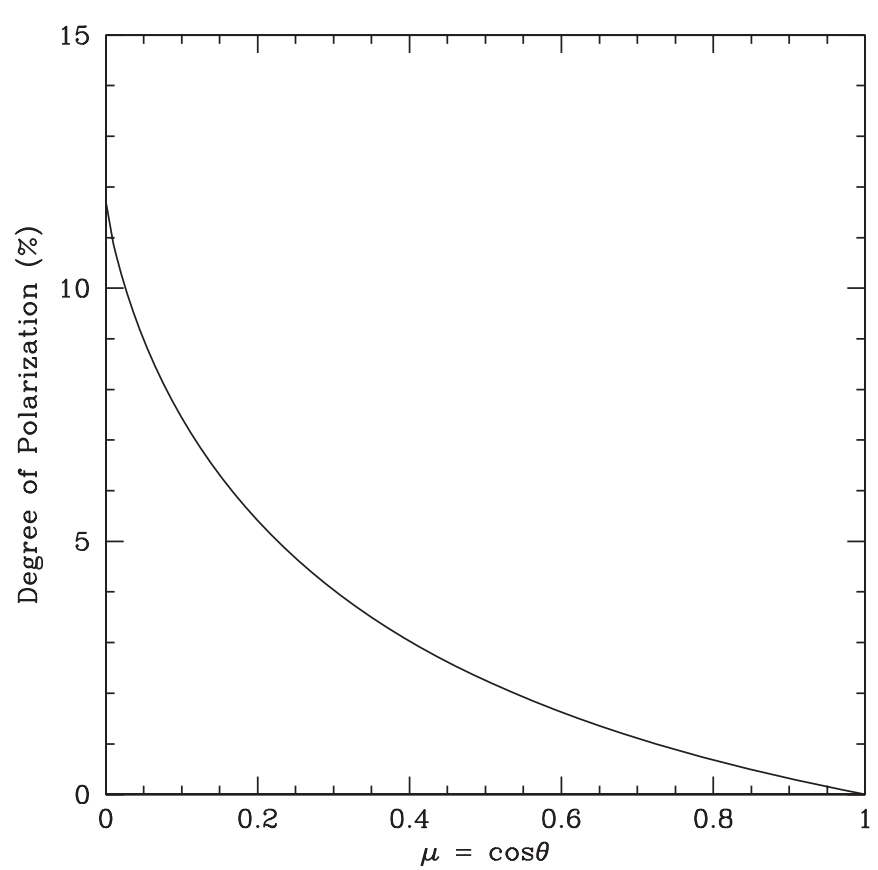

Figure 1. Degree of polarization as a function of $\mu=\cos \theta$ for a semi-infinite scattering atmosphere, where $\theta$ is the angle between the line of sight and the normal to the plane of the atmosphere.

where $\alpha_{n}$ and $A_{n}$ are the moments of order $n$ of $H_{l}(\mu)$ and $H_{r}(\mu)$,

$$
\begin{gathered}
\alpha_{n} \equiv \int_{0}^{1} H_{l}(\mu) \mu^{n} d \mu, \\
A_{n} \equiv \int_{0}^{1} H_{r}(\mu) \mu^{n} d \mu(n \geqslant 0) .
\end{gathered}
$$

Figure 1 shows the degree of polarization $P$ as a function of $\mu$ for a scattering atmosphere. At $\mu=1(\theta=0)$, we have $P=0$ because of symmetry. As $\mu$ decreases (i.e., $\theta$ increases), $P$ increases monotonically and reaches its maximum value of $11.7 \%$ at $\mu=0(\theta=\pi / 2)$. For a semi-infinite atmosphere we always have $I_{r}(\mu)>I_{l}(\mu)$ (see Table XXIV in Chandrasekhar 1960), except at $\mu=1(\theta=0)$ where $I_{r}(\mu)=I_{l}(0, \mu)$. Hence we have $\psi=\pi / 2$ for all $\mu$, where $\psi$ is the angle of the linear polarization vector relative to the meridian plane.

The above results are for a pure scattering semi-infinite atmosphere. However, an accretion disk also has absorption processes which may be important, especially at large disk radii where the temperature is low. Absorption tends to destroy the polarization of photons and hence to reduce the degree of polarization. We defer a detailed computation of the effect of absorption to a later paper. Here, following Laor et al. (1990) and Chen \& Eardley (1991), we simply reduce the degree of polarization by the following approximate factor

$$
q_{\mathrm{w}}=\frac{\tau_{\mathrm{es}}}{\tau_{\mathrm{es}}+\tau_{\mathrm{ab}}}
$$

where $\tau_{\mathrm{es}}$ is the optical depth due to electron scattering, and $\tau_{\mathrm{ab}}$ is the optical depth due to photon absorption. Thus, we write the degree of polarization of photons emerging from the disk surface as

$$
P_{\mathrm{em}}=q_{\mathrm{w}} P_{\mathrm{em}, 0},
$$

where $P_{\mathrm{em}, 0}$ is the degree of polarization for a pure electron scattering atmosphere and is given by Equations (1) and (2). We 
assume that the orientation of the polarization is not affected by absorption.

To complete the description of the polarized emission from an accretion disk, we need the flux $F$ and the optical depths $\tau_{\text {es }}$ and $\tau_{\mathrm{ab}}$ as functions of the radius $R$. These are obtained using the disk model described in Appendix A.

\section{POLARIZATION OF THE DISK EMISSION AS OBSERVED BY A REMOTE OBSERVER}

An arbitrary radiation field can be decomposed into an unpolarized component, with Stokes parameters

$$
\{(1-P) I, 0,0,0\},
$$

and a completely polarized component, with Stokes parameters

$$
\{P I, Q, U, V\} \text {, }
$$

where $P$ is the degree of polarization. Further we have

$$
\begin{gathered}
\tan 2 \psi=\frac{U}{Q}, \\
\sin 2 \beta=\frac{V}{\left(Q^{2}+U^{2}+V^{2}\right)^{1 / 2}},
\end{gathered}
$$

where $\psi$ is the angle between the major axis of the polarization ellipse and the direction described by the subscript $l$ (see Section 3$),{ }^{3}$ and $\tan \beta$ is the ratio of the minor axis to the major axis of the polarization ellipse. Photons emitted by the disk are linearly polarized, so we have $V=0$ and $\beta=0$. Then, by the definition of the degree of polarization $P$, we have

$$
\begin{gathered}
Q=I_{\mathrm{p}} \cos 2 \psi, \\
U=I_{\mathrm{p}} \sin 2 \psi, \\
I_{\mathrm{p}} \equiv P I .
\end{gathered}
$$

Equivalently, we can write

$$
Q+i U=I_{\mathrm{p}} e^{2 i \psi} .
$$

When several independent streams of light are combined, the Stokes parameters for the combined radiation are the sums of the respective Stokes parameters of the individual streams (Chandrasekhar 1960); we refer to this as the superposition theorem. Therefore, we may consider the unpolarized and polarized components of the radiation separately.

For the polarized component, consider a beam of perfectly polarized radiation emitted by an infinitesimal surface element on the disk and received by an observer along an infinitesimal solid angle element $d \Omega_{\mathrm{obs}}$. Let this radiation have intensity $I_{\mathrm{p}, \mathrm{obs}}$ and polarization angle $\psi_{\mathrm{obs}}$ as measured by the observer. Then, the observed Stokes parameters are

$$
\left\{I_{\mathrm{p}, \mathrm{obs}}, Q_{\mathrm{obs}}, U_{\mathrm{obs}}, 0\right\},
$$

where

$$
\begin{aligned}
& Q_{\mathrm{obs}}=I_{\mathrm{p}, \mathrm{obs}} \cos 2 \psi_{\mathrm{obs}}, \\
& U_{\mathrm{obs}}=I_{\mathrm{p}, \mathrm{obs}} \sin 2 \psi_{\mathrm{obs}} .
\end{aligned}
$$

\footnotetext{
3 We denote the angle of the plane of polarization from the direction $l$ by $\psi$ (rather than the $\chi$ in Chandrasekhar 1960) to be consistent with Connors et al. (1980).
}

Summing over the radiation received from all disk elements we have, by the superposition theorem,

$$
\left\langle I_{\mathrm{p}, \mathrm{obs}}\right\rangle=\frac{1}{\Delta \Omega_{\mathrm{obs}}} \int I_{\mathrm{p}, \mathrm{obs}} d \Omega_{\mathrm{obs}},
$$

and

$$
\begin{aligned}
\left\langle Q_{\mathrm{obs}}\right\rangle+i\left\langle U_{\mathrm{obs}}\right\rangle & =\frac{1}{\Delta \Omega_{\mathrm{obs}}} \int\left(Q_{\mathrm{obs}}+i U_{\mathrm{obs}}\right) d \Omega_{\mathrm{obs}} \\
& =\frac{1}{\Delta \Omega_{\mathrm{obs}}} \int I_{\mathrm{p}, \mathrm{obs}} e^{2 i \psi_{\mathrm{obs}}} d \Omega_{\mathrm{obs}},
\end{aligned}
$$

where " \langle\rangle " denotes an average over solid angle, and $\Delta \Omega_{\mathrm{obs}}$ is the total solid angle subtended by the disk on the sky.

Note that, in general, we have

$$
\left\langle Q_{\text {obs }}\right\rangle^{2}+\left\langle U_{\text {obs }}\right\rangle^{2}<\left\langle I_{\mathrm{p}, \mathrm{obs}}\right\rangle^{2},
$$

i.e., the radiation is not fully polarized. Even though the individual contributions from each disk element may be perfectly polarized, the radiation received by the observer can still become partially polarized through averaging because the individual rays may have different values of $\psi_{\text {obs }}$ at the observer. In the context of an accretion disk around a black hole, even if all the emitted rays have the same polarization direction at their points of emission, geodesic propagation can cause changes in $\psi$ along each photon trajectory and cause a reduction in the observed degree of polarization. In other words, the rotation of the disk and the spin of the black hole can alter the polarization state of the original radiation and destroy the observed polarization at some level. In contrast, whatever unpolarized radiation is emitted by the disk remains unpolarized at the observer; thus, geodesic propagation and averaging can only decreased the degree of polarization.

For the unpolarized component, we have

$$
\left\langle I_{\mathrm{u}, \mathrm{obs}}\right\rangle=\frac{1}{\Delta \Omega_{\mathrm{obs}}} \int I_{\mathrm{u}, \mathrm{obs}} d \Omega_{\mathrm{obs}},
$$

where $I_{\mathrm{u}, \mathrm{obs}} \equiv I_{\mathrm{obs}}-I_{\mathrm{p}, \mathrm{obs}}$. By Equations (14) and (17) we have

$$
\begin{aligned}
\left\langle I_{\mathrm{u}, \mathrm{obs}}\right\rangle+\left\langle I_{\mathrm{p}, \mathrm{obs}}\right\rangle & =\frac{1}{\Delta \Omega_{\mathrm{obs}}} \int\left(I_{\mathrm{u}, \mathrm{obs}}+I_{\mathrm{p}, \mathrm{obs}}\right) d \Omega_{\mathrm{obs}} \\
& =\frac{1}{\Delta \Omega_{\mathrm{obs}}} \int I_{\mathrm{obs}} d \Omega_{\mathrm{obs}}=\left\langle I_{\mathrm{obs}}\right\rangle,
\end{aligned}
$$

i.e., the total intensity is conserved.

Using the fact that $I_{E_{\mathrm{loc}}} / E_{\mathrm{loc}}^{3}$ is invariant along the path of a photon, where $E_{\text {loc }}$ is the photon energy measured by a local observer along the photon path (Misner et al. 1973), Equation (15) can be rewritten as (see Equation (9) of Connors et al. 1980)

$$
\left\langle Q_{\mathrm{obs}}\right\rangle+i\left\langle U_{\mathrm{obs}}\right\rangle=\frac{1}{\Delta \Omega_{\mathrm{obs}}} \int g^{3} P_{\mathrm{em}} I_{\mathrm{em}} e^{2 i \psi_{\mathrm{obs}}} d \Omega_{\mathrm{obs}},
$$

where $P_{\mathrm{em}}$ is the degree of polarization of the radiation at the time that the radiation emerges from the disk surface, and $g$ is the redshift factor of the photon ( $\mathrm{Li}$ et al. 2005). Similarly, we can rewrite the total intensity as ${ }^{4}$

$$
\left\langle I_{\mathrm{obs}}\right\rangle=\frac{1}{\Delta \Omega_{\mathrm{obs}}} \int g^{3} I_{\mathrm{em}} d \Omega_{\mathrm{obs}} .
$$

\footnotetext{
4 Note that, in order to simplify the notation, we have suppressed the dependence on $E_{\mathrm{em}}$ - the photon energy as measured by a local observer corotating with the disk-in our expressions. This should cause no ambiguity.
} 
The observed average degree of polarization is then given by

$$
\langle P\rangle=\frac{1}{\left\langle I_{\mathrm{obs}}\right\rangle} \sqrt{\left\langle Q_{\mathrm{obs}}\right\rangle^{2}+\left\langle U_{\mathrm{obs}}\right\rangle^{2}},
$$

while the observed average angle of polarization, $\langle\psi\rangle$, is determined by ${ }^{5}$

$$
\begin{aligned}
\sin (2\langle\psi\rangle) & =\frac{\left\langle U_{\mathrm{obs}}\right\rangle}{\sqrt{\left\langle Q_{\mathrm{obs}}\right\rangle^{2}+\left\langle U_{\mathrm{obs}}\right\rangle^{2}}}, \\
\cos (2\langle\psi\rangle) & =\frac{\left\langle Q_{\mathrm{obs}}\right\rangle}{\sqrt{\left\langle Q_{\mathrm{obs}}\right\rangle^{2}+\left\langle U_{\mathrm{obs}}\right\rangle^{2}}} .
\end{aligned}
$$

In the definitions of the above dimensionless quantities the total solid angle $\Delta \Omega_{\text {obs }}$ cancels out.

The solution to Equation (22) is

$$
\langle\psi\rangle=\langle\psi\rangle_{\mathrm{pr}}+n \pi,
$$

where $n$ is any integer, and the primitive angle $\langle\psi\rangle_{\mathrm{pr}}$ (defined to be in the range $0-\pi$ ) is given by

$$
\langle\psi\rangle_{\mathrm{pr}}=\left\{\begin{array}{ll}
\frac{1}{2} \arccos \xi_{Q}, & \left(\left\langle U_{\mathrm{obs}}\right\rangle>0 ; 0<\langle\psi\rangle_{\mathrm{pr}}<\frac{\pi}{2}\right) \\
\pi-\frac{1}{2} \arccos \xi_{Q}, & \left(\left\langle U_{\mathrm{obs}}\right\rangle<0 ; \frac{\pi}{2}<\langle\psi\rangle_{\mathrm{pr}}<\pi\right)
\end{array},\right.
$$

where

$$
\xi_{Q} \equiv \frac{\left\langle Q_{\mathrm{obs}}\right\rangle}{\sqrt{\left\langle Q_{\mathrm{obs}}\right\rangle^{2}+\left\langle U_{\mathrm{obs}}\right\rangle^{2}}}
$$

In Equation (19), the degree of polarization $P_{\mathrm{em}}$ is evaluated at the disk surface by Equation (6), but the angle of polarization $\psi_{\text {obs }}$ is evaluated at the observer and is related to the angle at the disk surface by the propagation equation of the polarization vector. The propagation equation is described in detail in Appendix B.

In the limit of a semi-infinite plane atmosphere, the polarization vector of the emerging radiation is in the disk plane, so we have $\psi_{\mathrm{em}}=\pi / 2$ (see Section 3 ). Since $\psi_{\mathrm{obs}}$ appears in Equation (19) in the form of $\cos 2 \psi_{\mathrm{obs}}$ and $\sin 2 \psi_{\mathrm{obs}}$, the addition of $n \pi$ to $\psi_{\text {obs }}(n= \pm 1, \pm 2, \ldots)$ does not change the final results. Hence, at the observer, by Equation (B74) we can write

$$
\psi_{\mathrm{obs}}=\frac{1}{2} \pi-\Phi_{\mathrm{GR}},
$$

where $\Phi_{\mathrm{GR}}$ is calculated by Equations (B69), (B70), And (B75) as described in Appendix B.

Relativistic effects on photon polarization are reflected in the above formula in two aspects. First, relativistic effects lead to a rotation to the plane of polarization by an angle $\Phi_{\mathrm{GR}}$. Second, because of light bending the angle of the photon wavevector from the disk normal as the photon leaves the disk surface, $\theta$, differs from the disk inclination angle, $i_{\text {disk }}$. The Newtonian limit is obtained by setting $\Phi_{\mathrm{GR}}=0$ and $\theta=i_{\text {disk }}$. By Equation (26)

\footnotetext{
5 We do not use $\tan (2\langle\psi\rangle)=\left\langle U_{\text {obs }}\right\rangle /\left\langle Q_{\text {obs }}\right\rangle$. This single equation cannot determine the value of $\langle\psi\rangle$, since $\tan (2\langle\psi\rangle)$ is unchanged under the transformation $\left\langle Q_{\mathrm{obs}}\right\rangle \rightarrow-\left\langle Q_{\mathrm{obs}}\right\rangle$ and $\left\langle U_{\mathrm{obs}}\right\rangle \rightarrow-\left\langle U_{\mathrm{obs}}\right\rangle$. This is related to the fact that the primitive period of $\sin x($ and $\cos x)$ is $2 \pi$ while the primitive period of $\tan x$ is $\pi$.
}

we then have $\psi_{\mathrm{obs}}=\pi / 2$. Then, by Equation (19) we have $\left\langle U_{\text {obs }}\right\rangle=0$ and

$$
\left\langle Q_{\mathrm{obs}}\right\rangle=-\frac{P_{\mathrm{em}, 0}}{\Delta \Omega_{\mathrm{obs}}} \int q_{\mathrm{w}} g^{3} I_{\mathrm{em}} d \Omega_{\mathrm{obs}}
$$

where Equation (6) has been used, and $P_{\mathrm{em}, 0}=P_{\mathrm{em}, 0}\left(\theta=i_{\mathrm{disk}}\right)$. Hence, in the Newtonian limit we have $\langle\psi\rangle=\pi / 2$, and

$$
\langle P\rangle=P_{\mathrm{em}, 0} \frac{\int q_{\mathrm{w}} g^{3} I_{\mathrm{em}} d \Omega_{\mathrm{obs}}}{\int g^{3} I_{\mathrm{em}} d \Omega_{\mathrm{obs}}} .
$$

The high-energy spectrum is dominated by photons emitted by the inner disk region where photon absorption is not important $\left(q_{\mathrm{w}} \approx 1\right)$, hence we have $\langle P\rangle \approx P_{\mathrm{em}, 0}$ for high-energy photons.

As we will see in the next section, relativistic effects make the observed features of photons emitted by the disk dramatically different from that predicted by the Newtonian theory.

\section{APPLICATIONS TO THE MEASUREMENT OF BLACK HOLE SPIN}

\subsection{Comparison with Connors et al. (1980)}

Since the present work is a modern update on the pioneering work of Connors et al. (1980), we begin by recomputing the models discussed in their paper. Appendix B describes in detail how we calculate the polarization angle of an infinitesimal photon stream as observed by an observer at infinity. A key quantity is the primitive rotation angle $\Phi_{\mathrm{GR}}$ defined by Equations (B69), (B70), and (B75). The calculation of $\Phi_{\mathrm{GR}}$ requires an evaluation of the dimensionless quantities $\tilde{X}, \tilde{Y}, \tilde{S}$, and $\tilde{T}$ (Equations (B79)-(B81)), in addition to the orientation of the photon wavevector relative to the disk plane $(\theta$ and $\phi)$, the photon redshift factor $g$, and the Lorentz factor $\Gamma$ of the disk fluid. All these quantities can be evaluated with the formulae given in Appendix B of this paper and Appendix $\mathrm{C}$ of $\mathrm{Li}$ et al. (2005).

The polarization angle $\psi_{\text {obs }}$ is related to the rotation angle $\Phi_{\mathrm{GR}}$ by Equation (26). After calculating the angle $\psi_{\mathrm{obs}}$ for each light ray that reaches the observer within an infinitesimal solid angle $d \Omega_{\mathrm{obs}}$, one can evaluate the integrals in Equations (19) and (20) with the ray-tracing technique described in $\mathrm{Li}$ et al. (2005). Then the net degree of polarization $\langle P\rangle$ and angle of polarization $\langle\psi\rangle$ of the combined radiation, as seen by the observer, can be calculated via Equations (21) and (23)-(25). In the calculations reported here, the effect of photon absorption has been taken into account with the simple approach outlined in Section 3. That is, photon absorption reduces the degree of polarization by introducing a multiplying factor $q_{\mathrm{w}}$ defined in Equation (5).

Figure 2 shows the variation of the observed polarization angle $\langle\psi\rangle$ and the observed degree of polarization $\langle P\rangle$ as a function of photon energy for two disk inclinations $i_{\text {disk }}$ and various values of the dimensionless spin parameter $a_{*}=$ $c J / G M^{2}$, where $J$ is the angular momentum of the black hole. All the models shown in Figure 8 of Connors et al. (1980) are included here; several other models are also shown for completeness. Qualitatively, our results are similar to those obtained by Connors et al. (1980). Quantitatively, however, there are unexpectedly large differences. Compared with Figure 8 of Connors et al. (1980), our results in Figure 2 predict less photon absorption at low photon energies (indeed this can be 

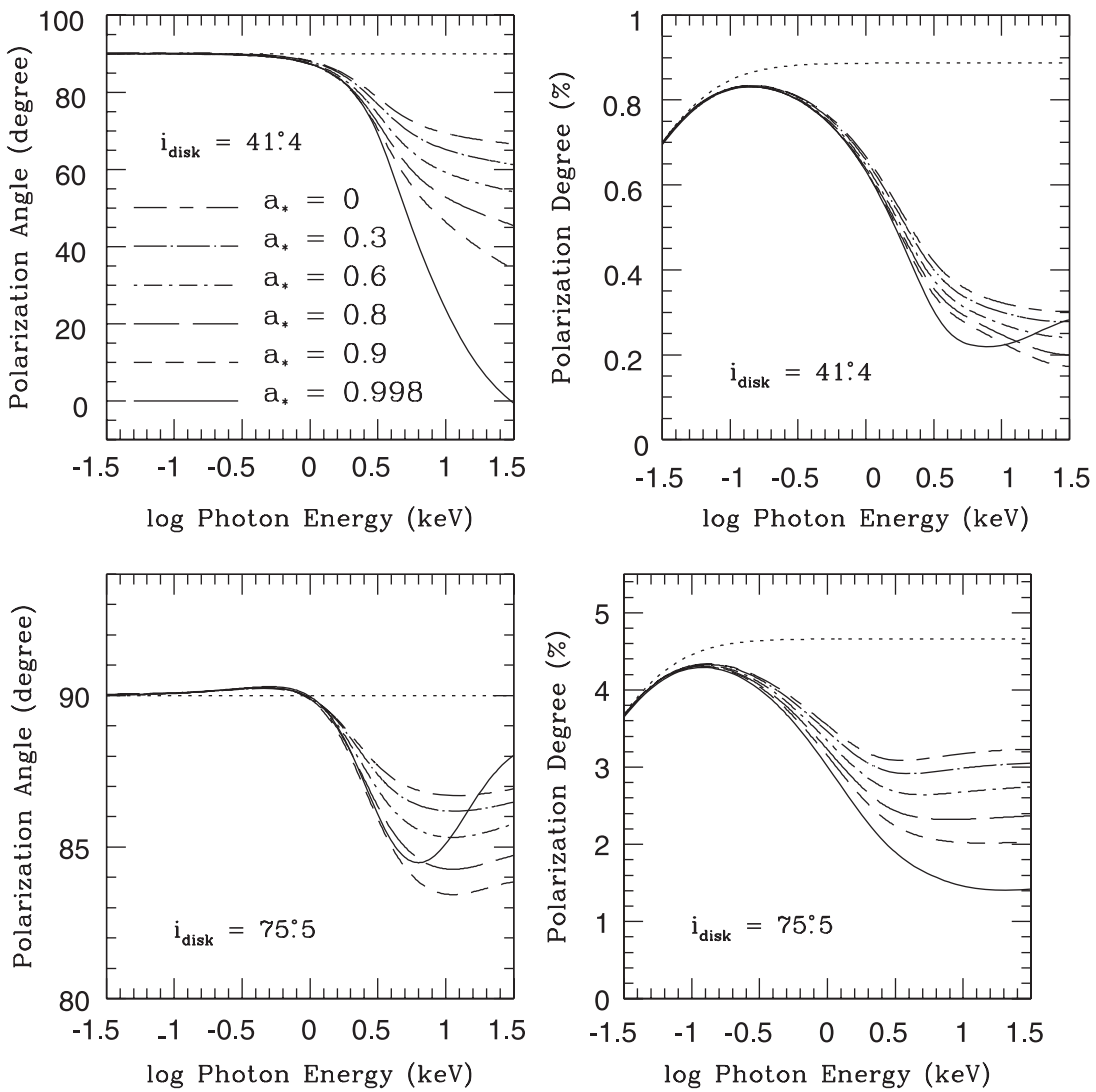

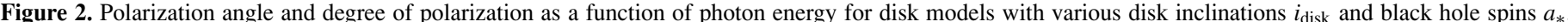

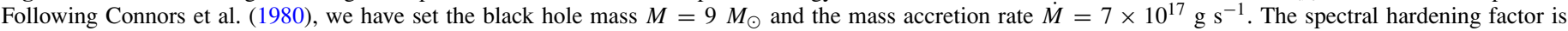

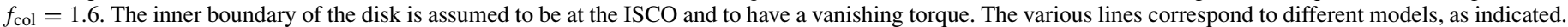

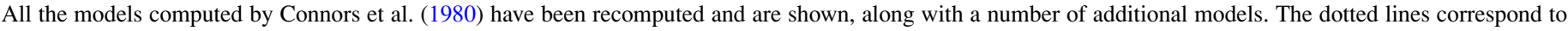
the Newtonian limit.

seen clearly in the Newtonian limit, the dotted lines in the figure). The results for high photon energy are also different. ${ }^{6}$

Rather surprisingly, the models differ considerably even in the basic shape of the continuum spectrum, as seen by comparing Figure 7 of Connors et al. (1980) with the corresponding results from our model shown in Figure 3. We have assumed that the disk emission is purely blackbody, and we have allowed for the spectral hardening due to electron scattering and Comptonization through a constant hardening factor $f_{\mathrm{col}}$. The spectra shown by Connors et al. do not appear to be pure blackbody (see their Figure 7). Indeed, their model seems to have too much emission both at low and high energies. ${ }^{7}$ Since we do not have sufficient information on the precise assumptions made by Connors et al. (1980) in their calculations, we are unable to resolve the discrepancy.

We have also compared our results with the calculations reported by Agol \& Krolik (2000) and Dovčiak et al. (2008). The

\footnotetext{
6 Note that we have set $\dot{M}=7 \times 10^{17} \mathrm{~g} \mathrm{~s}^{-1}$ in the models shown in Figures 2 and 3, since this is the value mentioned by Connors et al. (1980) in the second sentence of Section IVc. In their caption to their Figure 5, however, they mention $\dot{M}=10^{17} \mathrm{~g} \mathrm{~s}^{-1}$. We have computed models with the latter value of $\dot{M}$ (not shown) and the results are nearly the same.

7 At low photon energies, the blackbody photon flux density should have the well-known asymptotic form $N_{E} \propto E^{-2 / 3}$, e.g., our Figure 3, or equivalently the energy spectrum should go as $F_{E} \propto E^{1 / 3}$. However, Figure 7 of Connors et al. (1980) seems to behave as $N_{E} \propto E^{-1}$ near $E=0.1 \mathrm{keV}$. Also, their model $\mathrm{C}$, which corresponds to a rapidly spinning black hole $\left(a_{*}=0.998\right)$, has an unphysically hard spectrum with emission extending up to $100 \mathrm{keV}$, which is much too hot for thermal emission from a thin accretion disk around a stellar-mass black hole.
}

easiest and most direct comparison is when returning radiation and photon absorption are neglected, which has been assumed in the model of Dovčiak et al. (2008), and corresponds to the dashed lines in Figure 11 of Agol \& Krolik (2000). For this case, we find very good agreement with the results from our code for all the quantities of interest: flux, degree of polarization, and polarization angle. When returning radiation is included, Agol \& Krolik (2000) calculate the polarization induced by scattering off the surface of the disk. This effect is currently neglected in our model. We also neglect the effect of Faraday rotation due to magnetic fields in the disk atmosphere (Agol et al. 1998).

Before concluding this subsection, we would like to note the following interesting point. Figure 2 shows that, for a fixed $M$ and $i_{\text {disk }}$, the polarization of the observed radiation exhibits a large variation as a function of the spin parameter. Therefore, if we have already measured $M$ and $i_{\text {disk }}$ (see the next two subsections), then we could use polarization data to constrain $a_{*}$. This would provide an estimate of $a_{*}$ independent of the disk continuum fitting method. In fact, with sufficiently high-quality polarization data we might even be able to solve for both $i_{\text {disk }}$ and $a_{*}$ without using the continuum spectrum at all.

\subsection{Spin-Inclination Degeneracy in the Disk Continuum Spectrum}

We proceed now to discuss the continuum-fitting method and the important role that polarization measurements could play in this method. To determine the spin parameter $a_{*}$ of a black hole in an X-ray binary, we fit the X-ray continuum 


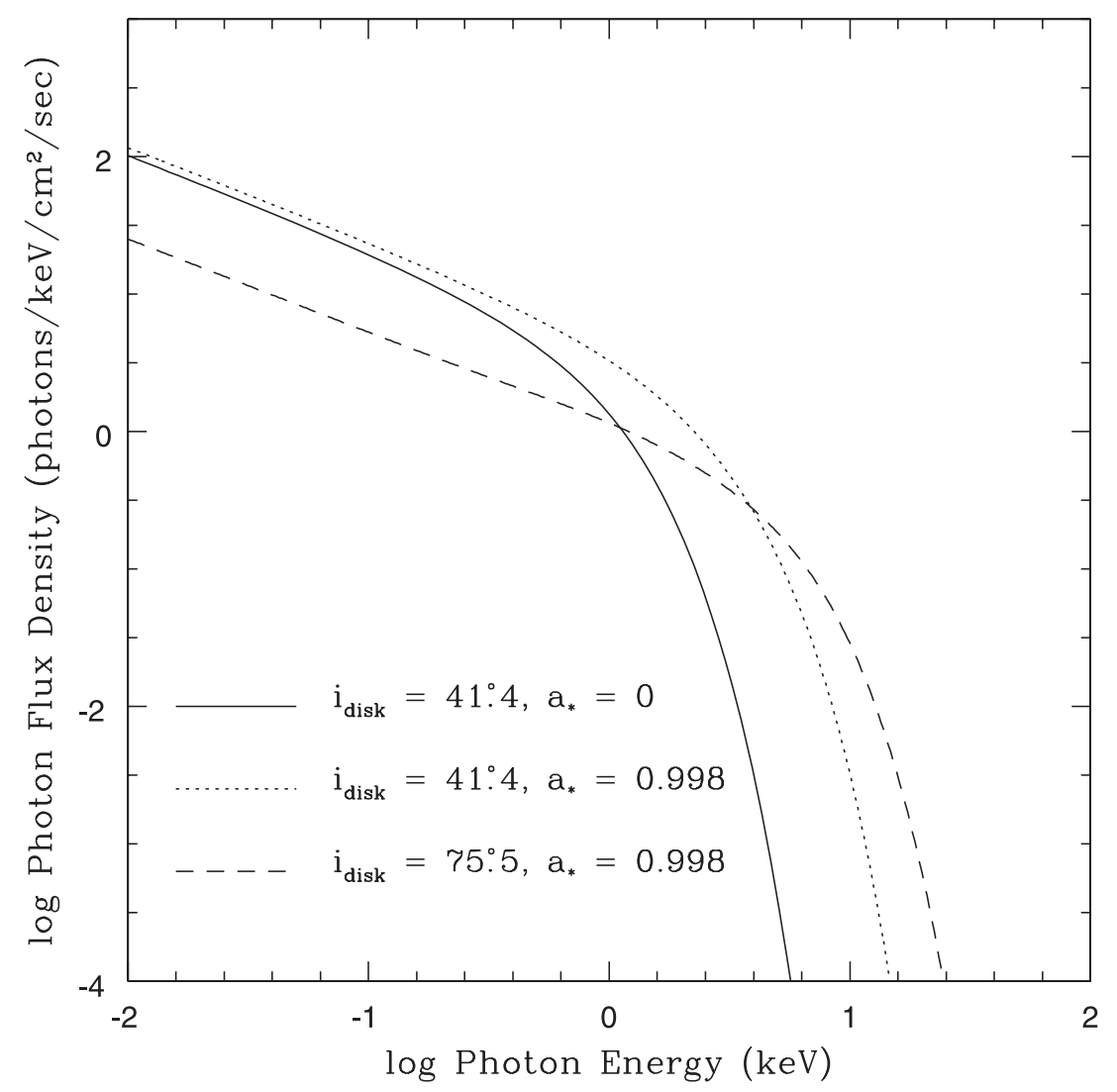

Figure 3. Continuum disk spectra computed with the publicly available relativistic accretion disk model KERRBB (Li et al. 2005) for the three disk models shown in Figure 7 of Connors et al. (1980). The distance to the source is assumed to be $10 \mathrm{kpc}$ and the spectral hardening factor is taken to be 1.6.

spectrum of its accretion disk using the publicly available model KERRBB. Three parameters of the system need to be measured independently: the mass of the black hole $M$, the distance to the black hole $D$, and the disk inclination angle $i_{\text {disk }}$. KERRBB has two principal fit parameters: the disk mass accretion rate $\dot{M}$ and the dimensionless spin parameter $a_{*}$. The mass accretion rate serves the role of a normalization factor and depends on the X-ray luminosity, while $a_{*}$ is determined principally by the temperature or hardness of the X-ray spectrum. Generally, it is straightforward to estimate these two parameters from X-ray continuum data. In fact, even when the spectral data require additional features for a good fit, e.g., photoelectric absorption, an iron line, or a weak Comptonized tail, one can still estimate $\dot{M}$ and $a_{*}$ with good precision.

In addition to $M, D$, and $i_{\text {disk }}$, the spin determination requires a theoretical model of the disk flux profile $F(R)$ as a function of radius $R$, and a detailed disk atmosphere model to estimate the spectral hardening factor $f_{\text {col }}$. For $F(R)$, KERRBB makes use of the relativistic disk model of Novikov \& Thorne (1973). The no-torque inner boundary condition assumed in this model has been questioned (e.g., Krolik 1999; Krolik \& Hawley 2002; Krolik et al. 2005), but recent work suggests that it may not be a serious issue when the disk luminosity is low and the disk is geometrically thin (Paczyński 2000; Li 2002; Afshordi \& Paczyński 2003; Shafee et al. 2008a, 2008b). With regard to $f_{\text {col }}$, disk atmosphere models including metal opacities have been recently computed and $f_{\text {col }}$ has been estimated as a function of the disk luminosity and inclination (Davis et al. 2005; Davis \& Hubeny 2006; Davis et al. 2006). This is a significant improvement over previous models which included only freefree opacity (Shimura \& Takahara 1995).
We thus expect that a reliable determination of $a_{*}$ of a stellar black hole can be made when we have high-quality X-ray data in the thermal state-usually not a problem-when we have good measurements of $M, D$, and $i_{\text {disk }}$. For a number of stellar black holes, $M$ and $D$ are well measured (Charles \& Coe 2006; Orosz 2003; Orosz et al. 2007). However, the inclination angle of the inner disk cannot be directly measured, except in a few cases when the system has a radio jet and the orientation of the jet (which is presumably perpendicular to the inner disk plane) can be determined (Hjellming \& Rupen 1995; Hjellming et al. 2000; Orosz et al. 2001). For the remaining systems, what we can measure is the inclination of the binary orbit $i_{\text {orb }}$, and we have to assume that the inner disk is aligned with the binary orbital plane: $i_{\text {disk }}=i_{\text {orb }}$. This assumption is probably reasonable (see Section 2.2), but it would be better if we could avoid it altogether.

In view of the uncertainty in the disk inclination, could we obtain $i_{\text {disk }}$ directly from X-ray spectral data? Unfortunately, this is not possible since a given observed continuum spectrum can be fitted well with different combinations of $a_{*}$ and $i_{\text {disk}}$. Table 1 shows an example of a set of degenerate models with distinct combinations of $a_{*}$ and $i_{\text {disk }}$. The mass of the black hole is fixed at $10 M_{\odot}$, the distance to the black hole is fixed at 10 $\mathrm{kpc}$, the spectral hardening factor is assumed to be fixed at 1.6, and the mass accretion rate $\dot{M}$ of each model has been adjusted such that all the models have the same luminosity. Figure 4 shows the disk continuum spectra of these models. Although the models have very different values of $a_{*}$ and $i_{\text {disk }}$, we see that their continuum spectra are nearly indistinguishable. Therefore, the spectrum alone is insufficient to solve for $i_{\text {disk }}$. As we now show, polarization can very effectively break the degeneracy. 


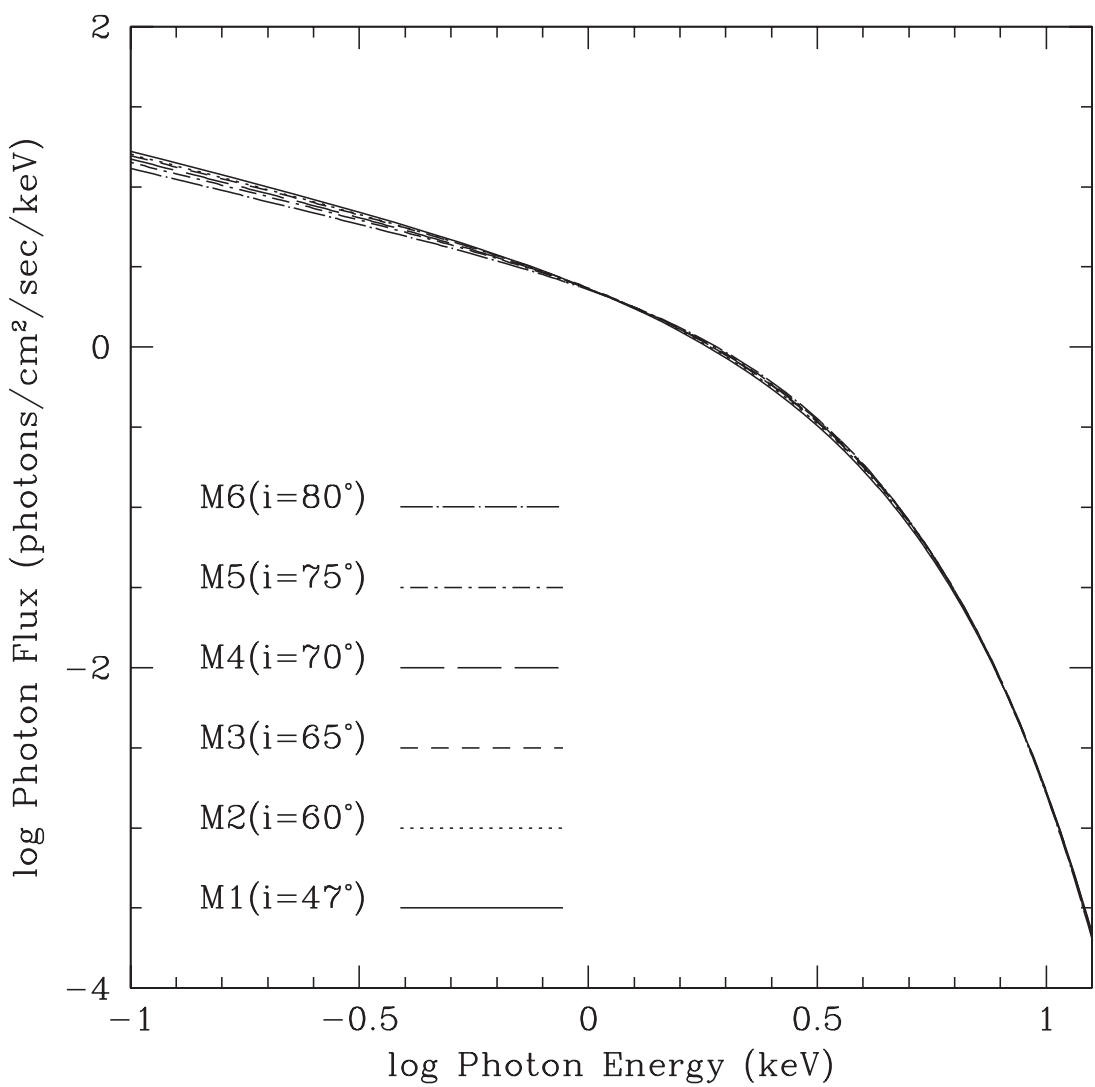

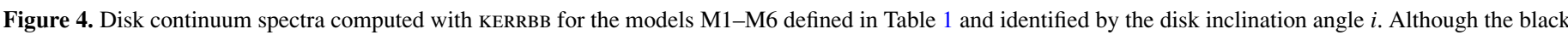

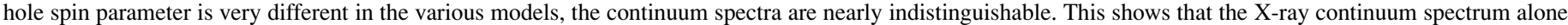
cannot be used to determine both the spin parameter and the disk inclination.

Table 1

Parameters Corresponding to the Models Shown in Figures 4-6

\begin{tabular}{lccc}
\hline \hline Model & $a_{*}$ & $i_{\text {disk }}$ & $\dot{M}$ \\
\hline M1 & 0.998 & 47.0 & 0.48 \\
M2 & 0.900 & 60.0 & 1.00 \\
M3 & 0.830 & 65.0 & 1.40 \\
M4 & 0.750 & 70.0 & 2.00 \\
M5 & 0.630 & 75.0 & 3.20 \\
M6 & 0.450 & 80.0 & 5.80 \\
\hline
\end{tabular}

Notes. Explanation of symbols: $a_{*}$, dimensionless spin parameter of the black hole; $i_{\text {disk }}$, inclination angle of the disk in degrees; $\dot{M}$, mass accretion rate in units of $10^{18} \mathrm{~g} \mathrm{~s}^{-1}$. Other parameters: mass of the black hole is $10 M_{\odot}$; distance to the black hole is $10 \mathrm{kpc}$; spectral hardening factor is 1.6. The inner boundary of the disk is at the ISCO and is assumed to have a zero torque boundary condition.

\subsection{Resolving the Spin-Inclination Degeneracy via Polarization Measurements}

Figure 5 shows the degree of polarization $\langle P\rangle$ as a function of photon energy for the degenerate models defined in Table 1. We see that $\langle P\rangle$ depends sensitively on the disk inclination. For the six models in Table 1, the disk inclination angle increases monotonically from model M1 to model M6, and we see that the degree of polarization of the disk radiation also increases monotonically. The difference in $\langle P\rangle$ between the extreme models is quite large.

Figure 6 shows the corresponding results for the polarization angle $\langle\psi\rangle$. Once again we see a large variation as a function of disk inclination. The variations are especially dramatic at photon energies on the order of several keV. Since the thermal emission from the disk peaks in this region of the spectrum (and photoelectric absorption is unlikely to be important), the effect could be easily measured (see Section 6).

These results demonstrate that the degeneracy between the black hole spin and the disk inclination in the continuum blackbody spectrum of the accretion disk can be resolved by polarization measurements. Note that the degree of polarization, in particular, shows large variations with disk inclination. To illustrate this fact, we show in Figure 7 the run of $\langle P\rangle$ with photon energy for an accretion disk with $\dot{M}=2 \times 10^{18} \mathrm{~g} \mathrm{~s}^{-1}$ (similar to model M4 in Table 1) and a range of inclination angles $i_{\text {disk }}$ around a black hole of mass $M=10 M_{\odot}$ and spin parameter $a_{*}=0.75$. Over the range $30^{\circ} \leqslant i_{\text {disk }} \leqslant 80^{\circ}$, we see that $\langle P\rangle$ varies by about an order of magnitude. Thus, an observation of the polarization would provide an accurate determination of the inclination of the inner disk. A fit of the continuum spectrum would then give an accurate measurement of the spin of the black hole.

As a byproduct, such work would resolve the question of whether or not the inner disk in a black hole X-ray binary is aligned with the orbital plane (see Section 2.2). Knowing the answer to this question would provide valuable constraints on models of core collapse and stellar black hole formation.

\section{THE PROSPECTS FOR AN OBSERVATIONAL TEST}

Is the inner accretion disk around stellar black holes warped away from the orbital plane (Section 2.2)? If so, is the warping severe enough to compromise the estimates of spin obtained 


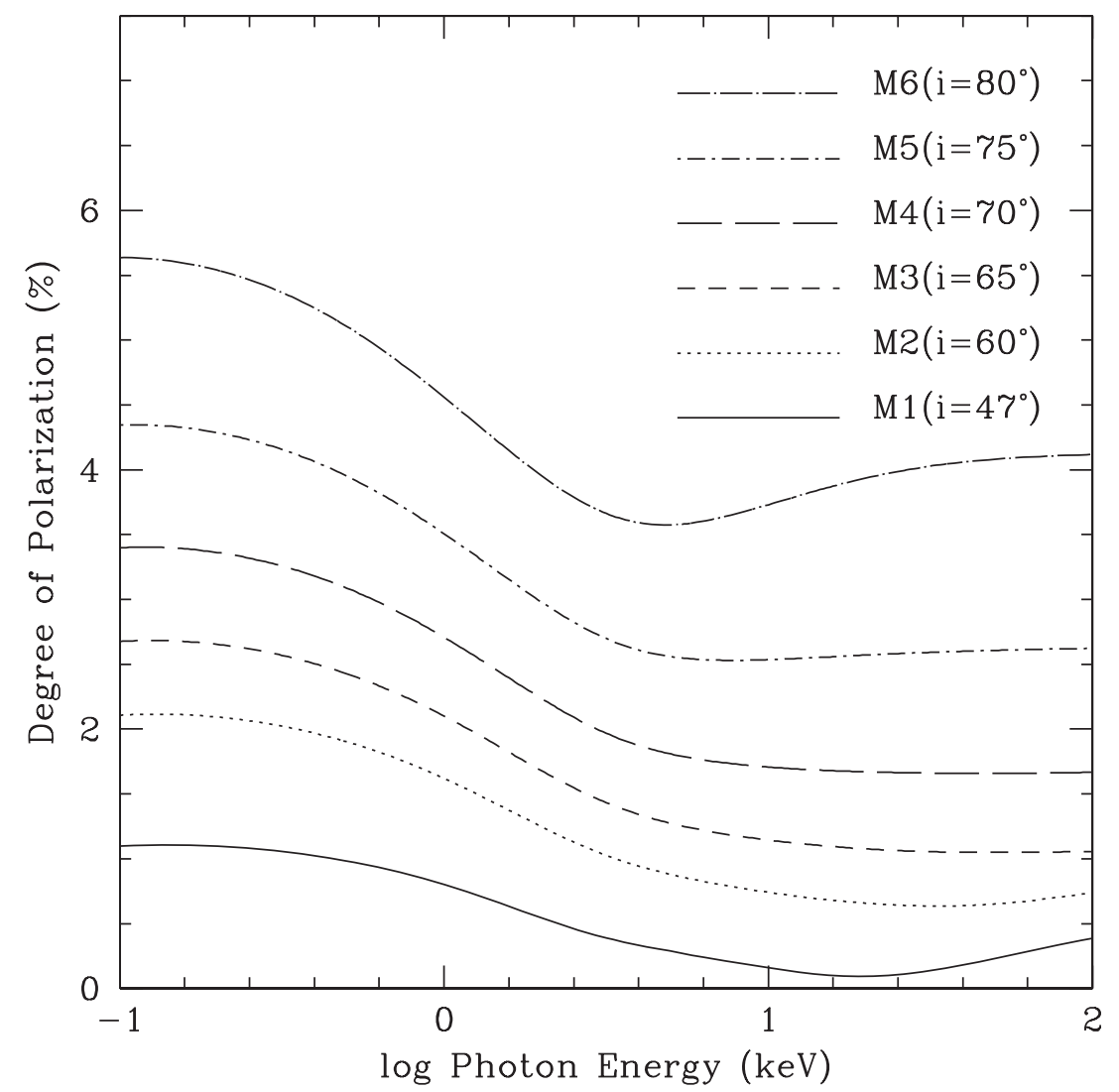

Figure 5. Degree of polarization vs. photon energy for the set of degenerate disk models M1-M6 defined in Table 1 and identified by the disk inclination angle $i$. The computations were done using an extended version of KERRBB. In contrast to Figure 4, where the continuum spectra of these models were identical, here the models are seen to have widely different degrees of polarization.

using the continuum-fitting method (Sections 2.1 and 5.2)? In this section, we show schematically that we can expect definitive answers to these question in the near future through polarization measurements in the X-ray band.

$\mathrm{X}$-ray polarimetry is presently a hot topic, as evidenced by the 2004 X-ray Polarimetry Workshop at Stanford University ${ }^{8}$ and the upcoming conference to be held in Rome: The Coming Age of X-ray Polarimetry. ${ }^{9}$ Furthermore, several polarimetric missions have been proposed recently. We now briefly consider the performance of two very different instrument concepts developed within the severe constraints of a NASA Small Explorer (SMEX)-class payload. These are modest instruments because the mission costs for a SMEX are capped at $\$ 105$ million (excluding the launch vehicle).

The instrument considered by a team at the Goddard Space Flight Center (GSFC) is a broadband $(1-10 \mathrm{keV})$ polarimeter, which measures the angular distribution of the tracks of the photoelectrons (Costa et al. 2001; Swank et al. 2004). The other instrument concept was developed by a team at the Smithsonian Astrophysical Observatory (SAO). It is a narrowband, Braggcrystal instrument, which operates at $2.6 \mathrm{keV}$ and $5.2 \mathrm{keV}$ (E. Silver 2008, private communication) and is a modern version of the crystal polarimeter flown aboard OSO-8 (Weisskopf et al. 1978). The sensitivity of these two instruments is nearly identical and is summarized in Table 2. As indicated in the table, either instrument can, for example, detect polarization in a 1 Crab source at the $0.3 \%$ level in 1 day and at the $0.1 \%$ level in 10 days.

\footnotetext{
8 http://heasarc.gsfc.nasa.gov/docs/heasarc/polar/polar.html

$9 \mathrm{http}: / /$ projects.iasf-roma.inaf.it/xraypol/
}

Table 2

Minimum Detectable Polarization $(\%)^{\mathrm{a}}$

\begin{tabular}{lcccc}
\hline \hline Intensity $(\mathrm{Crab})$ & 0.001 & 0.01 & 0.1 & 1 \\
\hline$T_{\text {obs }}=1$ days & 10 & 3 & 1 & 0.3 \\
$T_{\text {obs }}=10$ days & 3 & 1 & 0.3 & 0.1 \\
\hline
\end{tabular}

Note.

${ }^{\text {a }}$ Nominal sensitivities accurate to $\approx 20 \%$ (Swank et al. 2004; E. Silver 2008, private communication).

Our focus here is the TD state of black hole binaries (Section 2.1), or an intermediate or steep power-law (SPL) state with a strong thermal component (McClintock \& Remillard 2006), which we here refer to as a near-TD state. The dominance in this state of the thermal component makes it ideally suited for the determination of the inclination of the inner disk via polarimetry. Fortunately, nearly all of the many blackhole transient sources display a TD spectrum for weeks at a time during their outburst cycle. Furthermore, the sources are bright during this thermal phase, which makes them feasible polarimetric targets. Table 3 lists a selected sample of more than 40 known transient and persistent black hole systems (McClintock \& Remillard 2006; Remillard \& McClintock 2006; Orosz et al. 2007; Silverman \& Filippenko 2008). The average intensities and outburst durations are restricted to those times when the source was in the TD or near-TD state. These data are only crude characterizations of the actual intensities/durations; see the references for precise information.

As the data in Table 3 indicate, many sources have maintained a TD spectrum with an intensity of $\sim 1$ Crab for several tens of 


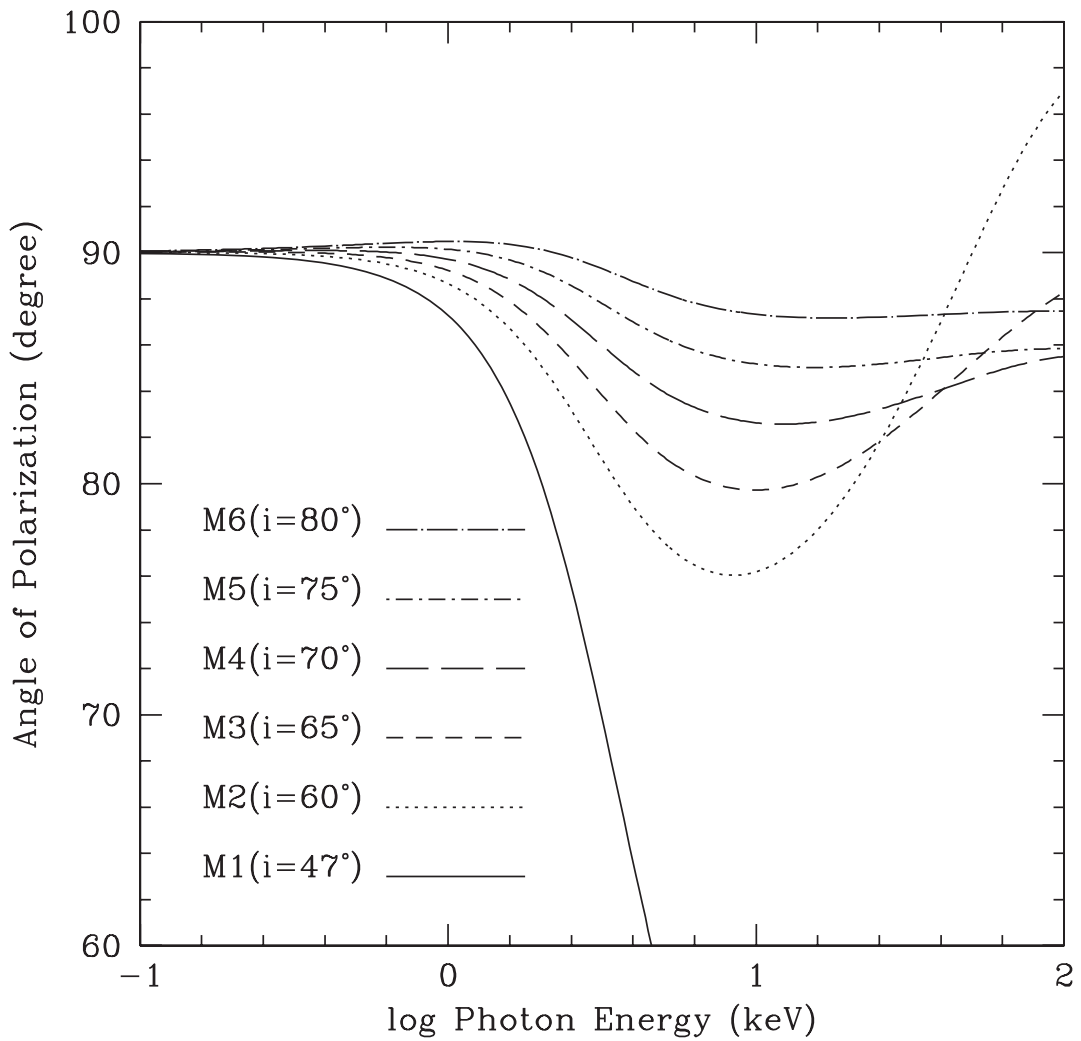

Figure 6. Similar to Figure 5, but showing the angle of polarization. Once again the models are easily distinguishable.

Table 3

Selected Black Hole Binaries

\begin{tabular}{|c|c|c|c|c|c|c|}
\hline & $\begin{array}{c}\text { Coordinate } \\
\text { Name }\end{array}$ & $\begin{array}{c}\text { Common } \\
\text { Name/Prefix }^{\mathrm{a}}\end{array}$ & $\begin{array}{c}\text { Year/ } \\
\text { No. of Outbursts }\end{array}$ & $\begin{array}{c}\text { Intensity } \\
\left(\mathrm{Crab}^{\mathrm{c}}\right)\end{array}$ & $\begin{array}{c}\text { Outburst }^{\mathrm{d}} \\
\text { Duration (d) }\end{array}$ & References ${ }^{\mathrm{e}}$ \\
\hline 1 & $0620-003$ & (A) & $1975 / 1$ & 15 & 100 & Matilsky et al. (1976) \\
\hline 2 & $1124-684$ & Nova Mus 91 & $1991 / 1$ & 1 & 100 & Ebisawa et al. (1994) \\
\hline 3 & $1543-475$ & $(4 U)$ & $1971 / 4$ & 1 & 100 & RM06 \\
\hline 4 & $1550-564$ & (XTE J) & $1998 / 5$ & 2 & 100 & RM06 \\
\hline 5 & $1650-500$ & (XTE J) & $2001 / 1$ & 0.5 & 100 & MR06 \\
\hline 6 & $1655-40$ & $(\mathrm{GRO} J)$ & $1994 / 3$ & 1 & 300 & RM06 \\
\hline 7 & $1659-487$ & GX 339-4 & $1972 / 12$ & 0.5 & 200 & RM06 \\
\hline 8 & $1705-250$ & Nova Oph 77 & $1977 / 1$ & 1 & 50 & Watson et al. (1978) \\
\hline 9 & $1859+226$ & (XTE J) & $1999 / 1$ & 0.5 & 100 & RM06 \\
\hline 11 & $2000+251$ & (GS) & $1988 / 1$ & 3 & 100 & Tsunemi et al. (1989) \\
\hline 12 & $1630-472$ & $(4 U)$ & $1971 / 16$ & 0.2 & 100 & MR06 \\
\hline 13 & $1743-322$ & $(\mathrm{H})$ & $1977 / 4$ & 0.5 & 100 & RM06 \\
\hline 14 & $0538-641$ & LMC X-3 & Persistent & 0.03 & $\cdots$ & MR06 \\
\hline 15 & $0540-697$ & LMC X-1 & Persistent & 0.02 & $\cdots$ & MR06 \\
\hline 10 & $1915+105$ & (GRS) & Quasi-persistent $^{\mathrm{f}}$ & 1 & $\cdots$ & McClintock et al. (2006) \\
\hline 16 & $1956+350$ & Cyg X-1 & Persistent $^{\mathrm{g}}$ & 1 & $\cdots$ & Wilms et al. (2006) \\
\hline
\end{tabular}

Notes.

a A prefix to a coordinate name is enclosed in parentheses.

${ }^{\mathrm{b}}$ Crude estimate of average intensity in TD or near-TD state; see references.

c $1 \mathrm{Crab} \approx 2.8 \times 10^{-8} \mathrm{erg} \mathrm{cm}^{-2} \mathrm{~s}^{-1}(1-10 \mathrm{keV})$ for a Crab-like spectrum with $\Gamma=2.1$.

${ }^{\mathrm{d}}$ Crude estimate of time in TD or near-TD state; see references.

e RM06, Remillard \& McClintock 2006; MR06, McClintock \& Remillard 2006.

${ }^{\mathrm{f}}$ In outburst since discovery in 1992; occasionally observed in TD state (McClintock et al. 2006).

g Observed in a soft, near-TD state for extended periods (e.g., Wilms et al. 2006).

days. Therefore, a modest instrument like those described above would have the capability to measure the predicted polarizations of up to $\sim 5 \%$ (Figure 7 ) with a sensitivity of $\sim 0.1 \%$ for several transient (and persistent) sources during the course of a mission. Furthermore, polarization measurements of this precision could in many cases be repeated several times during the $\sim 50-100$ days thermal decay phase of a typical transient source.

Even a single $\sim 1-10$ days observation would allow the inclination of the inner disk, for a wide range of inclinations, to be determined to roughly $1^{\circ}$ (E. Silver 2008, private communication). Meanwhile the inclination angle $i_{\text {orb }}$ of the orbital plane 


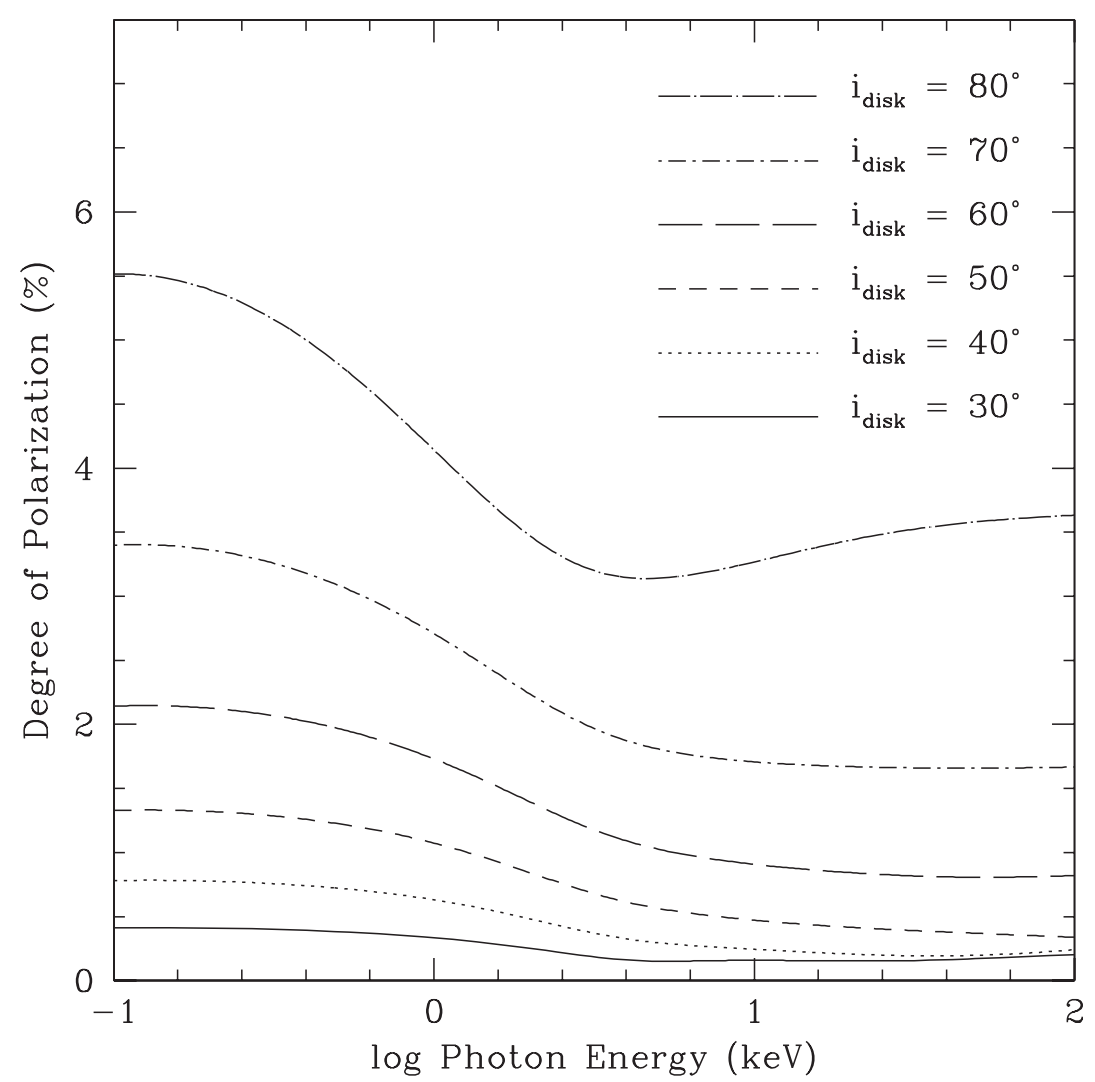

Figure 7. Degree of polarization vs. photon energy for a series of accretion disk models with varying inclination angle $i_{\text {disk }}$. All the models have $\dot{M}=2 \times 10^{18} \mathrm{~g} \mathrm{~s}{ }^{-1}$, $M=10 M_{\odot}$, and $a_{*}=0.75$. The large variation in the degree of polarization with $i_{\text {disk }}$ means that it should be possible to determine the disk inclination accurately with polarization data.

is already known for most of the sources in Table 3 to a few degrees (Charles \& Coe 2006; Orosz 2003). Thus, a simple comparison of the values of $i_{\text {disk }}$ and $i_{\text {orb }}$ for a source in question will provide a stringent test of whether its disk is warped.

\section{DISCUSSION AND CONCLUSIONS}

In order to secure the measurement of black hole spin using the continuum-fitting method, it is essential to obtain an independent determination of the inclination of the inner accretion disk $i_{\text {disk }}$. We have shown that this appears to be entirely feasible via X-ray polarimetry. Our models predict polarizations of up to $\sim 5 \%$ that vary monotonically with inclination, while sensitivities of $\sim 0.1 \%$ are achievable with quite modest SMEX-class polarimeters. Such instruments are expected to be capable of routinely making measurements of $i_{\text {disk }}$ over a wide range of inclinations with a precision of $\sim 1^{\circ}$. Meanwhile, with full attention it will be possible to achieve measurements of similar quality for the orbital inclination angle $i_{\text {orb }}$, given recent advances in optical/nearIR instrumentation (e.g., Vernet et al. 2007) and adaptive optics (e.g., van Dam et al. 2006). Once reliable values of these two inclination angles have been obtained for a sample of black hole binaries, the question will simply be: is $i_{\text {disk }} \approx i_{\text {orb }}$, or is it not?

There are of course significant hurdles that must be cleared. For example, the model of the disk atmosphere presented here includes only an approximate treatment of the effects of absorption. In the manner of Davis \& Hubeny (2006), one must model the non-LTE effects, Compton scattering, and the opacities due to ions of the abundant elements. Magnetohydrodynamic (MHD) models of thin disks in general relativity, which include radiation and polarization, are the ultimate theoretical goal, and progress is now being made on this front (Beckwith et al. 2008; Noble et al. 2008; Reynolds \& Fabian 2008; Shafee et al. 2008a). Then, there are observational complications to consider. For example, even in the TD state there exists some remnant scattered coronal emission, which must be modeled. Some other examples of possible nettlesome sources of polarization include self-irradiation and reflection in the disk, scattering by interstellar grains, and the effects of a global component of magnetic field in the disk.

Clearly, the greatest hurdle is getting an X-ray polarimeter into space. Thirty years have passed since the tiny $O S O-8$ crystal instrument successfully measured the polarization of the Crab Nebula at $2.6 \mathrm{keV}$ to be $19.2 \% \pm 1.0 \%$ at a position angle of $156^{\circ} .4 \pm 1.4$ (Weisskopf et al. 1978). It is surely now time to open the polarimetric channel for serious exploration and discovery. In this paper, we have shown how a polarimeter can secure the measurement of black hole spin. This is just one exciting application of X-ray polarimetry and there are numerous others.

In the study of black holes, polarimetry furthermore promises to define the geometry of the emitting elements and constrain source models in decisive ways (Section 1). This is true, for instance, for modeling the relativistic iron line (Miller 2007), or relativistic jets (Mirabel \& Rodríguez 1999), or the mysterious power-law component that extends unbroken to $\mathrm{MeV}$ energies (Grove et al. 1998), or the global oscillations that sometimes modulate up to one quarter of the total accretion power (McClintock \& Remillard 2006). In modeling these and other black-hole phenomena, a central question is the geometry of the Comptonizing coronal source, which is vaguely and variously described as a sphere or a slab or a lamp post. Polarimetry will provide the best, and often only, clue to 
the actual geometry of the corona and other key structures (Mészáros et al. 1988; Blandford et al. 2002).

This work was supported in part by NASA grants NNH07ZDA001N and NNX08AJ55G and NSF grant AST0805832. We thank Eric Silver and Bill Forman for providing information on their instrument concept and Martin Elvis for a discussion on AGNs.

\section{APPENDIX A}

\section{RELATIVISTIC THIN ACCRETION DISK MODEL}

We make use of a simple $\alpha$-viscosity model to describe the disk (Shakura \& Sunyaev 1973), modified to include relativistic effects (Novikov \& Thorne 1973; Page \& Thorne 1974). Below, we first discuss the relativistic terms and then proceed to describe the disk model.

\section{A.1. The Disk Height}

By Equation (A8) of McClintock et al. (2006), the vertical gravitational acceleration in the comoving frame of the fluid, for small excursions $z$ from the disk midplane, is given by

$$
g_{z}=\xi \Omega_{\mathrm{K} 0}^{2} z
$$

where

$$
\Omega_{\mathrm{K} 0} \equiv \sqrt{\frac{G M}{R^{3}}}
$$

is the Newtonian angular velocity of the disk, $R$ is the cylindrical radius, and

$$
\begin{gathered}
\xi \equiv \frac{1}{\mathcal{C}}\left(1-4 a_{*} x^{-3}+3 a_{*}^{2} x^{-4}\right), \\
x \equiv \sqrt{\frac{R}{R_{\mathrm{g}}}}, \\
R_{\mathrm{g}} \equiv \frac{G M}{c^{2}} .
\end{gathered}
$$

The dimensionless function $\mathcal{C}$, and the functions $\mathcal{B}, \mathcal{D}$, and $\mathcal{Q}$ used later below, are defined in Novikov \& Thorne (1973) and Page \& Thorne (1974).

Hydrostatic equilibrium in the vertical direction gives

$$
\frac{c_{\mathrm{s}}^{2}}{H} \approx g_{z}(z=H)
$$

where $H$ is the height (vertical thickness) of the disk and $c_{\mathrm{s}}$ is the thermal sound speed of the gas at the disk midplane (both in the comoving frame). From Equations (A1) and (A4), we obtain

$$
H \approx \frac{c_{\mathrm{s}}}{\Omega_{\mathrm{V}}},
$$

where the vertical disk frequency

$$
\Omega_{\mathrm{V}} \equiv \Omega_{\mathrm{K} 0} \xi^{1 / 2}
$$

\section{A.2. The Flux Density and the Shear Stress of the Disk}

In this subsection, we set $G=c=1$ unless either of them or both appear explicitly in an expression. With these geometrized units, we have $R_{\mathrm{g}}=M$ and $x=(R / M)^{1 / 2}$.

Page and Thorne's functions $f$ and $\mathcal{Q}$ (Equations (15n) and (35) of Page \& Thorne 1974) are related by

$$
f=\frac{3}{2 M x^{4}} \frac{\mathcal{Q}}{\mathcal{B C}^{1 / 2}} .
$$

Then, by Equation (11b) of Page \& Thorne (1974) and Equation (D11) of Li et al. (2005), we have the disk flux density (see Equation (5.6.14b) of Novikov \& Thorne 1973)

$$
\begin{aligned}
F & =\frac{\dot{M}}{4 \pi R} f+\frac{g_{\text {in }}}{4 \pi R}\left(E^{\dagger}-\Omega L^{\dagger}\right)_{\text {in }}\left(-\frac{d \Omega}{d R}\right)\left(E^{\dagger}-\Omega L^{\dagger}\right)^{-2} \\
& =\frac{3 G \dot{M} M}{8 \pi R^{3}} \frac{1}{\mathcal{B C}^{1 / 2}}\left(\mathcal{Q}+\eta \epsilon_{\text {in }} \frac{\mathcal{C}_{\text {in }}^{1 / 2} x_{\text {in }}^{3}}{x \mathcal{B}^{-1} \mathcal{C}^{1 / 2}}\right)
\end{aligned}
$$

where $\Omega$ is the angular velocity of the disk, $E^{\dagger}$ and $L^{\dagger}$ are, respectively, the specific energy and the specific angular momentum of disk particles, $g_{\text {in }} \equiv \eta \epsilon_{\text {in }} \dot{M} / \Omega_{\text {in }} \geqslant 0$ is the torque applied at the inner boundary of the disk (at the ISCO), and $\epsilon_{\text {in }} \equiv 1-E_{\text {in }}^{\dagger}$ is the specific gravitational binding energy at the inner boundary (hereafter the subscript 'in' indicates evaluation at the disk inner boundary).

By Equation (11c) of Page \& Thorne (1974), the vertically integrated coordinate-frame component of the shear stress tensor of the disk is

$$
\begin{aligned}
W_{\varphi}^{R}=\frac{g}{2 \pi R} & =\frac{1}{2 \pi R}\left[\frac{E^{\dagger}-\Omega L^{\dagger}}{-d \Omega / d R} \dot{M} f+\frac{\left(E^{\dagger}-\Omega L^{\dagger}\right)_{\text {in }}}{E^{\dagger}-\Omega L^{\dagger}} g_{\text {in }}\right] \\
& =\frac{\dot{M}}{2 \pi}\left(\frac{M}{R}\right)^{1 / 2}\left(\mathcal{Q}+\eta \epsilon_{\text {in }} \frac{\mathcal{C}_{\text {in }}^{1 / 2} x_{\text {in }}^{3}}{x \mathcal{B}^{-1} \mathcal{C}^{1 / 2}}\right),
\end{aligned}
$$

where $\varphi$ is the azimuthal coordinate in the disk. The vertically integrated comoving-frame component of the shear stress, $W=$ $2 H t_{\hat{\varphi} \hat{R}}$ (the hats indicate coordinate components in a frame comoving with the disk), is related to $W_{\varphi}^{R}$ by (the last equation in page 428 of Novikov \& Thorne 1973)

$$
W_{\varphi}^{R}=R \mathcal{B C}^{-1 / 2} \mathcal{D} W
$$

Hence, Equation (A9) agrees with Equation (5.6.14a) of Novikov \& Thorne (1973) in the limit of $\eta=0$ (zero torque at the inner boundary). Then we have

$$
W=\frac{\dot{M}}{2 \pi}\left(\frac{M}{R^{3}}\right)^{1 / 2} \frac{\mathcal{C}^{1 / 2}}{\mathcal{B D}}\left(\mathcal{Q}+\eta \epsilon_{\text {in }} \frac{\mathcal{C}_{\text {in }}^{1 / 2} x_{\text {in }}^{3}}{x \mathcal{B}^{-1} \mathcal{C}^{1 / 2}}\right) .
$$

Following Shakura \& Sunyaev (1973), we describe the viscosity in the disk via a dimensionless parameter $\alpha$ and assume that $t_{\hat{\varphi} \hat{R}}=(3 / 2) \alpha P_{\mathrm{c}}$, where $P_{\mathrm{c}}$ is the pressure at the disk midplane, and the extra factor $3 / 2$ is in order to be similar to the Newtonian equation. Then we obtain

$3 H \alpha P_{\mathrm{c}}=\frac{\dot{M}}{2 \pi}\left(\frac{M}{R^{3}}\right)^{1 / 2} \frac{\mathcal{C}^{1 / 2}}{\mathcal{B D}}\left(\mathcal{Q}+\eta \epsilon_{\text {in }} \frac{\mathcal{C}_{\text {in }}^{1 / 2} x_{\text {in }}^{3}}{x \mathcal{B}^{-1} \mathcal{C}^{1 / 2}}\right)$. 
Using $2 H \alpha P_{\mathrm{c}}=2 H \alpha \rho c_{\mathrm{s}}^{2}=\alpha \Sigma c_{\mathrm{s}}^{2}$ and Equation (A2), where $\Sigma$ is the surface mass density of the disk, we then have

$$
3 \pi \frac{\alpha c_{\mathrm{s}}^{2}}{\Omega_{\mathrm{K} 0}} \Sigma=\dot{M} \frac{\mathcal{C}^{1 / 2}}{\mathcal{B} D}\left(\mathcal{Q}+\eta \epsilon_{\mathrm{in}} \frac{\mathcal{C}_{\mathrm{in}}^{1 / 2} x_{\mathrm{in}}^{3}}{x \mathcal{B}^{-1} \mathcal{C}^{1 / 2}}\right) .
$$

Note that the Newtonian limit $(x \gg 1)$ of equation (A13) is

$$
3 \pi \frac{\alpha c_{\mathrm{s}}^{2}}{\Omega_{\mathrm{K} 0}} \Sigma=\dot{M}\left[1-\left(\frac{R_{\mathrm{in}}}{R}\right)^{1 / 2}\right] .
$$

Although in this paper we only consider the case $\eta=0$, we keep $\eta$ in the above formulae for generality.

\section{A.3. One-Zone Disk Model}

We make the standard one-zone approximation to describe the vertical structure of the disk (Frank et al. 2002). Thus, in terms of the midplane pressure $P_{\mathrm{c}}$ and density $\rho_{\mathrm{c}}$, the thermal sound speed and the surface mass density are given by

$$
\begin{aligned}
c_{\mathrm{s}}^{2} & =P_{\mathrm{c}} / \rho_{\mathrm{c}}, \\
\Sigma & =2 H \rho_{\mathrm{c}} .
\end{aligned}
$$

To allow for the effect of scattering and absorption opacity, we use the following approximate formula to relate the escaping flux density $F$ to the midplane temperature $T_{\mathrm{c}}$,

$$
F=\frac{\sigma T_{\mathrm{c}}^{4}}{(3 / 4)\left[\tau / 2+1 / \sqrt{3}+1 /\left(3 \tau_{\mathrm{ab}}\right)\right]},
$$

where $\sigma$ is the Stefan-Boltzmann constant, $\tau_{\mathrm{ab}}$ is the absorptive optical depth from the midplane to the surface of the disk, and $\tau$ is the total optical depth. This formula is taken from Popham \& Narayan (1995) and is based on the work of Hubeny (1990). Correspondingly, we use the following equation of state of the fluid,

$$
P_{\mathrm{c}}=\frac{\rho_{\mathrm{c}} k T_{\mathrm{c}}}{\mu m_{\mathrm{u}}}+\frac{4 \sigma T_{\mathrm{c}}^{4}}{3 c}\left[\frac{\tau / 2+1 / \sqrt{3}}{\tau / 2+1 / \sqrt{3}+1 /\left(3 \tau_{\mathrm{ab}}\right)}\right],
$$

where the two terms correspond to gas and radiation pressure. Here, $k$ is the Boltzmann constant, $m_{\mathrm{u}}$ is the atomic mass unit, and $\mu$ is the mean molecular weight. We assume a fully ionized gas of solar composition, $\mu=0.603$, and we approximate $\tau$ and $\tau_{\mathrm{ab}}$ as

$$
\begin{array}{r}
\tau=\tau_{\mathrm{es}}+\tau_{\mathrm{ab}}, \quad \tau_{\mathrm{es}}=0.346(\Sigma / 2), \\
\tau_{\mathrm{ab}}=6.6 \times 10^{22} \rho_{\mathrm{c}} T_{\mathrm{c}}^{-7 / 2}(\Sigma / 2),
\end{array}
$$

where the coefficients are in cgs units.

The disk solution is easily obtained using the above equations. At a given radius $R$ and for an assumed value of $\Sigma$, Equation (A13) allows us to calculate $c_{\mathrm{s}}^{2}$, Equation (A5) gives $H$, Equation (A16) gives $\rho_{\mathrm{c}}$, Equation (A15) gives $P_{\mathrm{c}}$, Equations (A18) and (A19) allow us to solve for $T_{\mathrm{c}}$, and finally Equation (A17) gives the radiation flux $F$. Equating this estimate of the flux to the correct value given in (A8) gives an algebraic equation with only one unknown, $\Sigma$, which can be solved numerically. Once we have $\Sigma$, we immediately obtain the values of $\tau_{\mathrm{es}}$ and $\tau_{\mathrm{ab}}$ and this allows us to calculate the polarization suppression factor $q_{\mathrm{w}}$ in Equation (5).

\section{APPENDIX B}

\section{PROPAGATION OF THE POLARIZATION VECTOR IN KERR SPACETIME}

In this section, we use geometrized units: $G=c=1$.

In terms of the Newman-Penrose orthonormal (ON) tetrad $\left\{l^{a}, n^{a}, m^{a}, \bar{m}^{a}\right\}$ (where $l^{a}$ and $n^{a}$ are real vectors, $m^{a}$ and $\bar{m}^{a}$ are complex, and $\bar{m}^{a}=\left(m^{a}\right)^{*}$ is the complex conjugate of $\left.m^{a}\right)$, the Kerr metric tensor can be written as (Chandrasekhar 1983; Wald 1984) $)^{10}$

$$
g_{a b}=2\left[-l_{(a} n_{b)}+m_{(a} \bar{m}_{b)}\right],
$$

where the use of parentheses "()" in subscripts denotes symmetrization of a tensor. The $\mathrm{ON}$ tetrad satisfies the orthogonality conditions

$$
\mathbf{l} \cdot \mathbf{m}=\mathbf{l} \cdot \overline{\mathbf{m}}=\mathbf{n} \cdot \mathbf{m}=\mathbf{n} \cdot \overline{\mathbf{m}}=0,
$$

the normalization conditions

$$
\mathbf{l} \cdot \mathbf{n}=-1, \mathbf{m} \cdot \overline{\mathbf{m}}=1,
$$

as well as the null conditions

$$
\mathbf{l} \cdot \mathbf{l}=\mathbf{n} \cdot \mathbf{n}=\mathbf{m} \cdot \mathbf{m}=\overline{\mathbf{m}} \cdot \overline{\mathbf{m}}=0,
$$

where $\mathbf{I} \cdot \mathbf{m} \equiv l^{a} m_{a}$, etc. Then, for any vector $f^{a}$, we have

$$
\mathbf{f} \cdot \mathbf{f}=-2[(\mathbf{f} \cdot \mathbf{l})(\mathbf{f} \cdot \mathbf{n})-(\mathbf{f} \cdot \mathbf{m})(\mathbf{f} \cdot \overline{\mathbf{m}})] .
$$

Two Killing vectors and one Killing tensor in a Kerr spacetime lead to three conserved quantities along geodesics of particles and photons: the energy, the angular momentum about the symmetric axis of the black hole, and the square of the 'total angular momentum' (Carter 1968; Bardeen et al. 1972; Wald 1984). The three conserved quantities enable one to solve the timelike and null geodesics in a Kerr spacetime (Chandrasekhar 1983; Li et al. 2005).

The Kerr metric-like any other type D (II-II) vacuum spacetime-possesses a conformal Killing spinor (Walker \& Penrose 1970; Wald 1984) which enables one to determine the parallel propagation of polarization vectors along null geodesics in a simple manner. This is possible because of the WalkerPenrose theorem: if $\mathbf{k}$ is a null geodesic, affinely parameterized, and $\mathbf{f}$ is a vector orthogonal to $\mathbf{k}$ and parallelly propagated along it, then, in a type-D spacetime, the quantity

$$
K_{\mathrm{WP}}=2[(\mathbf{k} \cdot \mathbf{l})(\mathbf{f} \cdot \mathbf{n})-(\mathbf{k} \cdot \mathbf{m})(\mathbf{f} \cdot \overline{\mathbf{m}})] \Psi_{2}^{-1 / 3}
$$

is conserved along the geodesic (Walker \& Penrose 1970; Chandrasekhar 1983), i.e.,

$$
k^{a} \nabla_{a} K_{\mathrm{WP}}=0 .
$$

In Equation (B6), $\Psi_{2}$ is the only nonvanishing Weyl scalar in a Kerr spacetime

$$
\begin{aligned}
\Psi_{2} & =-\frac{M r}{|\rho|^{6}}\left(r^{2}-3 a^{2} \cos ^{2} \vartheta\right)-i \frac{a M \cos \vartheta}{|\rho|^{6}}\left(3 r^{2}-a^{2} \cos ^{2} \vartheta\right) \\
& =-\frac{M}{\rho^{* 3}},
\end{aligned}
$$

${ }^{10}$ We adopt the signature convention of Misner et al. (1973) and Wald (1984), which is opposite to that of Chandrasekhar (1983). 
where we have adopted Boyer-Lindquist coordinates $(t, r, \vartheta, \varphi)$, and

$$
|\rho|^{2} \equiv \rho \rho^{*}=r^{2}+a^{2} \cos ^{2} \vartheta
$$

Two corollaries can be derived from the Walker-Penrose theorem.

Corollary 1: $K_{\mathrm{WP}}$ is invariant under the transformation $\mathbf{f} \rightarrow$ $\mathbf{f}+\alpha \mathbf{k}$, where $\alpha$ is an arbitrary function.

Corollary 2: In a Kerr spacetime, the Walker-Penrose theorem implies that

$$
\left|K_{\mathrm{WP}}\right|^{2}=M^{-2 / 3}\left[\mathcal{Q}+\left(L_{z}-a E_{\infty}\right)^{2}\right](\mathbf{f} \cdot \mathbf{f}),
$$

where $E_{\infty}=-k_{t}$ is the conserved energy-at-infinity, $L_{z}=k_{\varphi}$ is the conserved angular momentum about the axis of the black hole, and $\mathcal{Q}=k_{\vartheta}^{2}+L_{z}^{2} \cot ^{2} \vartheta-a^{2} E_{\infty}^{2} \cos ^{2} \vartheta$. When $a=0$, $\mathcal{Q}+L_{z}^{2}$ is the square of the total angular momentum.

Proof of Corollary 1 is straightforward by applying the identity $\mathbf{k} \cdot \mathbf{k}=0$ and Equation (B5).

Proof of Corollary 2 is as follows. Following the proof of Corollary 2 in Section 60 of Chandrasekhar (1983), we have

$$
\left|K_{\mathrm{WP}}\right|^{2}=2\left|\Psi_{2}\right|^{-2 / 3}(\mathbf{k} \cdot \mathbf{m})(\mathbf{k} \cdot \overline{\mathbf{m}})(\mathbf{f} \cdot \mathbf{f}) .
$$

Then, defining

$$
\begin{aligned}
S & \equiv \frac{k_{\varphi}}{\sin \vartheta}+a \sin \vartheta k_{t}=\frac{L_{z}}{\sin \vartheta}-a \sin \vartheta E_{\infty}, \\
T & \equiv k_{\vartheta}=\operatorname{sign}\left(k_{\vartheta}\right) \sqrt{\mathcal{Q}+a^{2} E_{\infty}^{2} \cos ^{2} \vartheta-L_{z}^{2} \cot ^{2} \vartheta},
\end{aligned}
$$

one finds that

$$
(\mathbf{k} \cdot \mathbf{m})(\mathbf{k} \cdot \overline{\mathbf{m}})=\frac{1}{2|\rho|^{2}}\left(S^{2}+T^{2}\right) .
$$

It can be checked that

$$
S^{2}+T^{2}=\mathcal{Q}+\left(L_{z}-a E_{\infty}\right)^{2}=\text { constant }
$$

and

$$
\left|\Psi_{2}\right|^{-2 / 3}=M^{-2 / 3}|\rho|^{2}
$$

Substituting Equations (B14)-(B16) into Equation (B11), the identity in Equation (B10) is proved.

Let us define

$$
K_{\mathrm{WP}} \equiv(-M)^{-1 / 3}\left(K_{1}+i K_{2}\right)
$$

where $K_{1}$ and $K_{2}$ are real constants. Then, substituting Equation (B8) into Equation (B6), we obtain

$$
K_{1}+i K_{2}=2 \rho^{*}[(\mathbf{k} \cdot \mathbf{l})(\mathbf{f} \cdot \mathbf{n})-(\mathbf{k} \cdot \mathbf{m})(\mathbf{f} \cdot \overline{\mathbf{m}})] .
$$

In terms of vector components in the Boyer-Lindquist coordinate system, we have

$$
\begin{aligned}
K_{1}+i K_{2}= & \frac{1}{r+i a \cos \vartheta}\left\{\left(r^{2}+a^{2}\right)\left(k_{r} f_{t}-k_{t} f_{r}\right)\right. \\
& +a\left(k_{r} f_{\varphi}-k_{\varphi} f_{r}\right)+\frac{i}{\sin \vartheta}\left[k_{\vartheta} f_{\varphi}-k_{\varphi} f_{\vartheta}\right. \\
& \left.\left.-a \sin ^{2} \vartheta\left(k_{t} f_{\vartheta}-k_{\vartheta} f_{t}\right)\right]\right\}
\end{aligned}
$$

where we have used the fact that

$$
k_{a} f^{a}=0 \text {. }
$$

Equation (B19) is very useful since $k_{t}$ and $k_{\varphi}$ are conserved quantities, and $k_{\vartheta}$ can also be expressed in terms of conserved quantities $\left(E_{\infty}, L_{z}\right.$, and $\left.\mathcal{Q}\right)$. Therefore, $k_{r}$ can be calculated using the equation $g^{a b} k_{a} k_{b}=0$.

To solve for the propagation of photon polarization, we take $\mathbf{f}$ to be the unit polarization vector, i.e., $\mathbf{f}=\mathbf{A} / A$ where $\mathbf{A}$ is the wave amplitude vector (Misner et al. 1973). The vector f satisfies Equation (B20), and

$$
k^{a} \nabla_{a} f^{b}=0,
$$

which states that the polarization vector is parallelly propagated along rays. Although for arbitrarily polarized radiation $\mathbf{f}$ is complex, for a linearly polarized beam it can be chosen to be a real vector (Misner et al. 1973). Hence, we assume that $\mathbf{f}$ is real, with

$$
f_{a} f^{a}=1 .
$$

The four-vector $\mathbf{f}$ is only defined to within a multiple of $\mathbf{k}$ since $\mathbf{k}$ satisfies the geodesic equation. Under a transformation $\mathbf{f} \rightarrow \mathbf{f}^{\prime}=\mathbf{f}+\alpha \mathbf{k}$, it is obvious that Equations (B20) and (B22) are preserved. However, for $\mathbf{f}^{\prime}$ to be a solution of the parallel propagation Equation (B21), $\alpha$ must satisfy $k^{a} \nabla_{a} \alpha=0$, i.e., $\alpha$ must be a constant along the null geodesic.

Because of the Walker-Penrose theorem, we do not need to solve the propagation Equation (B21) of the polarization vector explicitly. Instead, Equations (B19) and (B20) can be used to determine the polarization vector. By Corollary 2, Equation (B22) can be derived from Equation (B19). Since $f^{a}$ is parallelly propagated along the geodesic of $k^{a}, f_{a} f^{a}$ and $f_{a} k^{a}$ are preserved along the geodesic. Hence, if at a point on the light ray Equations (B20) and (B22) are satisfied, they are satisfied everywhere on the light ray. However, Equation (B20) cannot be derived from the Walker-Penrose theorem so it is an independent equation.

Equation (B19) is equivalent to two real equations. Then, together with Equation (B20), we have three equations for the four components of $f^{a}$. The vector $f^{a}$ is determined up to an addition of a multiple of the wavevector $k^{a}$ (Corollary 1). However, this degree of freedom does not affect physical measurements, since electromagnetic waves are transverse waves and a multiple of $k^{a}$ only changes the component of $f^{a}$ along the light propagation direction and the local time direction (Misner et al. 1973, Exercise 22.12). Hence, an uncertainty arising from a multiple of $k^{a}$ does not prohibit us from making physical interpretations. In fact, we can make use of this gauge freedom to simplify the calculation by choosing a convenient form of $f^{a}$. Therefore, Equations (B19) and (B20) are sufficient for solving the physical components of $f^{a}$. After the evaluation of the integral constants $K_{1}$ and $K_{2}$, Equations (B19) and (B20) can be used to determine the component of $f^{a}$ at any point on the light ray.

Since we have assumed that $\mathbf{f} \cdot \mathbf{f}=1$, by Equations (B10), (B15), and (B17) we have

$$
K_{1}^{2}+K_{2}^{2}=S^{2}+T^{2}=\mathcal{Q}+\left(L_{z}-a E_{\infty}\right)^{2} .
$$

\section{B.1. Evaluation of $K_{1}$ and $K_{2}$ on the Disk Plane}

On the disk plane we have $\vartheta=\pi / 2,|\rho|^{2}=r^{2}$, and $\Psi_{2}=-M / r^{3}$. Equation (B19) is then reduced to

$$
K_{1}+i K_{2}=\left(r^{2}+a^{2}\right) A_{1}+a A_{2}+i\left(A_{3}+a A_{4}\right),
$$




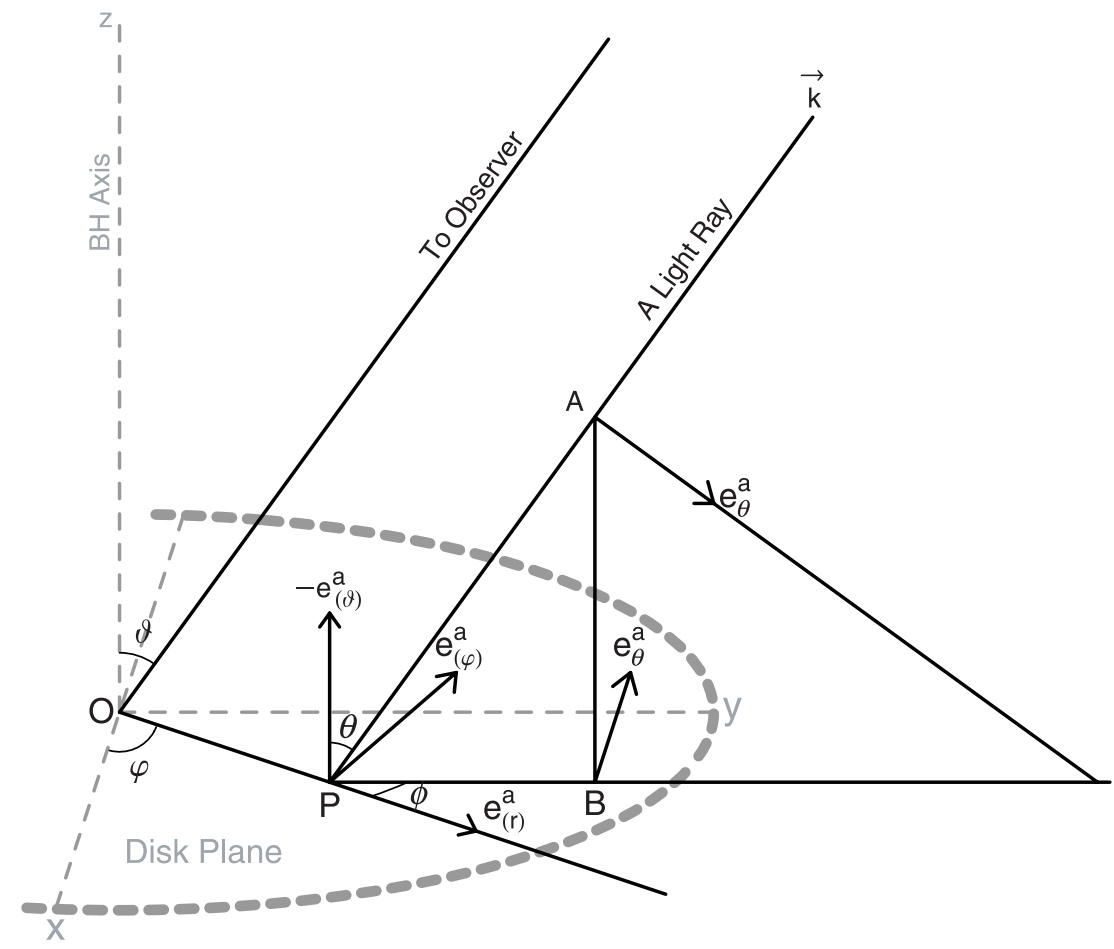

Figure 8. The coordinate system on the disk plane. The disk center is located at $O .\left\{e_{(t)}^{a}, e_{(r)}^{a}, e_{(\vartheta)}^{a}, e_{(\varphi)}^{a}\right\}$ is a local rest frame of the disk, with $e_{(t)}^{a}$ not shown. A light ray is emitted by a disk particle at $P$, propagates along a direction labeled by $\vec{k}$. The plane $P A B$ is a meridian plane at $P$, perpendicular to the disk plane. In the spherical coordinate system $(\theta, \phi), \theta$ is measured from the disk normal $-e_{(\vartheta)}^{a}$ in the meridian plane, and $\phi$ is measured from $e_{(r)}^{a}$ in the disk plane. The unit vectors $e_{\theta}^{a}$ (in the meridian plane and perpendicular to $\vec{k}$ ) and $e_{\phi}^{a}$ (in the disk plane and perpendicular to $P B$ ) are shown.

where

$$
\begin{aligned}
& A_{1}=r^{-1}\left(k_{r} f_{t}-k_{t} f_{r}\right), \\
& A_{2}=r^{-1}\left(k_{r} f_{\varphi}-k_{\varphi} f_{r}\right), \\
& A_{3}=r^{-1}\left(k_{\vartheta} f_{\varphi}-k_{\varphi} f_{\vartheta}\right), \\
& A_{4}=r^{-1}\left(k_{\vartheta} f_{t}-k_{t} f_{\vartheta}\right) .
\end{aligned}
$$

To evaluate the functions $A_{1}, \ldots, A_{4}$ in terms of the local parameters of photons as they leave the disk, e.g., the photon energy and the propagation direction as measured by an observer corotating with the disk, we will make use of two local frames: the locally nonrotating frame $\left\{e_{t}^{a}, \quad e_{r}^{a}, \quad e_{\vartheta}^{a}, e_{\varphi}^{a}\right\}$ which has a zero angular momentum, and the local rest frame

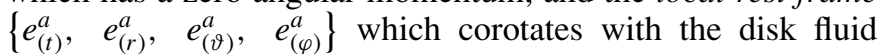
(Bardeen et al. 1972; Novikov \& Thorne 1973; Li et al. 2005). The two frames are related by a Lorentz transformation

$$
\begin{aligned}
e_{t}^{a} & =\Gamma\left[e_{(t)}^{a}-v_{\varphi} e_{(\varphi)}^{a}\right], \\
e_{\varphi}^{a} & =\Gamma\left[-v_{\varphi} e_{(t)}^{a}+e_{(\varphi)}^{a}\right], \\
e_{r}^{a} & =e_{(r)}^{a}, \\
e_{\vartheta}^{a} & =e_{(\vartheta)}^{a},
\end{aligned}
$$

where $v_{\varphi}$ is the azimuthal velocity of a disk particle relative to the locally nonrotating frame, and $\Gamma=\left(1-v_{\varphi}^{2}\right)^{-1 / 2}$ is the corresponding Lorentz factor.

The direction of the velocity of a photon as it crosses the disk plane is specified by the normalized four-wavevector of the photon, $n^{a} \equiv k^{a} / k^{(t)}=k^{a} / E_{\mathrm{loc}}$, where $k^{a}$ is the fourwavevector of the photon, and $E_{\mathrm{loc}}=k^{(t)}=-k_{a} e_{(t)}^{a}$ is the energy (frequency) of the photon measured in the local rest frame. The components of $n^{a}$ in the local rest frame of the disk are

$$
\begin{gathered}
n^{(t)}=1, \quad n^{(\vartheta)}=-\cos \theta, \\
n^{(r)}=\sin \theta \cos \phi, \quad n^{(\varphi)}=\sin \theta \sin \phi,
\end{gathered}
$$

where $(\theta, \phi)$ are spherical coordinates in the local rest frame, with the polar angle $\theta$ measured from the normal to the disk, and the azimuthal angle $\phi$ measured relative to the vector $e_{(r)}^{a}$ along the disk radial direction (see Figure 8). ${ }^{11}$

We write the polarization vector at the disk surface as

$$
\begin{aligned}
f^{a}= & f^{\theta} e_{\theta}^{a}+f^{\phi} e_{\phi}^{a}=\left(f^{\theta} \cos \theta \cos \phi-f^{\phi} \sin \phi\right) e_{(r)}^{a} \\
& +f^{\theta} \sin \theta e_{(\vartheta)}^{a}+\left(f^{\theta} \cos \theta \sin \phi+f^{\phi} \cos \phi\right) e_{(\varphi)}^{a} .
\end{aligned}
$$

By Equation (B33), the wavevector is given by

$$
k^{a}=E_{\mathrm{loc}}\left[e_{(t)}^{a}+\sin \theta \cos \phi e_{(r)}^{a}-\cos \theta e_{(\vartheta)}^{a}+\sin \theta \sin \phi e_{(\varphi)}^{a}\right]
$$

It can be checked that $k_{a} f^{a}=0$ is satisfied. The condition $f_{a} f^{a}=1$ implies that

$$
\left(f^{\theta}\right)^{2}+\left(f^{\phi}\right)^{2}=1 .
$$

Although with Equation (B34) the polarization vector is defined up to an addition of a multiple of $k^{a}$, Corollary 1 indicates that this does not affect the values of $K_{1}$ and $K_{2}$.

\footnotetext{
11 Note, by definition, $e_{\vartheta}^{a}$ points inward to the disk.
} 
The disk angular velocity $\Omega$ is related to the linear circular velocity $v_{\varphi}$ by

$$
\Omega=\omega_{0}+\chi_{0}\left(\frac{r^{2}}{A_{0}}\right)^{1 / 2} v_{\varphi}
$$

where $A_{0} \equiv r^{4}+a^{2} r(r+2 M), \chi_{0} \equiv\left(r^{2} \Delta / A_{0}\right)^{1 / 2}$ (the lapse function in the equatorial plane; $\left.\Delta \equiv r^{2}-2 M r+a^{2}\right)$, and $\omega_{0} \equiv 2 \mathrm{Mar} / A_{0}$ (the frame dragging angular velocity in the equatorial plane). The specific angular momentum and the specific energy of disk particles are respectively

$$
L^{\dagger}=\Gamma v_{\varphi}\left(\frac{A_{0}}{r^{2}}\right)^{1 / 2}, \quad E^{\dagger}=\Gamma \chi_{0}+\omega_{0} L^{\dagger}
$$

With the above equations and the definition of the locally nonrotating frame (Bardeen et al. 1972; Li et al. 2005), we obtain

$$
\begin{aligned}
A_{1}= & \frac{E_{\mathrm{loc}}}{\Delta^{1 / 2}}\left\{f^{\theta} E^{\dagger} \cos \theta \cos \phi-f^{\phi}\right. \\
& \left.\times\left[\Gamma \Omega\left(\frac{A_{0}}{r^{2}}\right)^{1 / 2} \sin \theta+E^{\dagger} \sin \phi\right]\right\}, \\
A_{2}= & \frac{E_{\mathrm{loc}}}{\Delta^{1 / 2}}\left\{-f^{\theta} L^{\dagger} \cos \theta \cos \phi+f^{\phi}\right. \\
& \left.\times\left[\Gamma\left(\frac{A_{0}}{r^{2}}\right)^{1 / 2} \sin \theta+L^{\dagger} \sin \phi\right]\right\}, \\
A_{3}= & -E_{\mathrm{loc}}\left\{f^{\theta}\left[L^{\dagger} \sin \theta+\Gamma\left(\frac{A_{0}}{r^{2}}\right)^{1 / 2} \sin \phi\right]\right. \\
& \left.+f^{\phi} \Gamma\left(\frac{A_{0}}{r^{2}}\right)^{1 / 2} \cos \theta \cos \phi\right\}, \\
A_{4}= & E_{\mathrm{loc}}\left\{f^{\theta}\left[E^{\dagger} \sin \theta+\Gamma \Omega\left(\frac{A_{0}}{r^{2}}\right)^{1 / 2} \sin \phi\right]\right. \\
+ & \left.f^{\phi} \Gamma \Omega\left(\frac{A_{0}}{r^{2}}\right)^{1 / 2} \cos \theta \cos \phi\right\} .
\end{aligned}
$$

Let us define

$$
\begin{aligned}
X \equiv & \frac{1}{\Gamma r}\left(\frac{A_{0}}{\Delta}\right)^{1 / 2}\left[\left(r^{2}+a^{2}\right) E^{\dagger}-a L^{\dagger}\right] \\
& =r^{2}+a^{2}-a \Delta^{1 / 2} v_{\varphi} \\
Y \equiv & \frac{A_{0}}{r^{2} \Delta^{1 / 2}}\left[\left(r^{2}+a^{2}\right) \Omega-a\right] \\
& =-a \Delta^{1 / 2}+\left(r^{2}+a^{2}\right) v_{\varphi}
\end{aligned}
$$

which satisfy

$$
X^{2}-Y^{2}=\frac{A_{0}}{\Gamma^{2}}
$$

Then by Equation (B24) we have

$$
K_{1}=E_{\mathrm{loc}} \frac{\Gamma r}{A_{0}^{1 / 2}}\left[\left(f^{\theta} \cos \theta \cos \phi-f^{\phi} \sin \phi\right) X\right.
$$

$$
\begin{aligned}
& \left.-f^{\phi} \sin \theta Y\right], \\
K_{2}= & -E_{\mathrm{loc}} \frac{\Gamma r}{A_{0}^{1 / 2}}\left[f^{\theta} \sin \theta Y\right. \\
& \left.+\left(f^{\theta} \sin \phi+f^{\phi} \cos \theta \cos \phi\right) X\right] .
\end{aligned}
$$

It can be checked that Equation (B23) is satisfied.

\section{B.2. The Limit at Infinity}

As $r \rightarrow \infty$, we have $\chi^{-1} \approx 1+M / r$, and $\omega \approx$ $2 M a / r^{3}$. Expansion of the locally nonrotating frame at infinity leads to

$$
\begin{aligned}
e_{t}^{a} \approx & \left(1+\frac{M}{r}\right)\left(\frac{\partial}{\partial t}\right)^{a}+\mathcal{O}\left(\frac{1}{r^{2}}\right)\left(\frac{\partial}{\partial t}\right)^{a} \\
& +\mathcal{O}\left(\frac{1}{r^{2}}\right) \frac{1}{r}\left(\frac{\partial}{\partial \varphi}\right)^{a}, \\
e_{r}^{a} \approx & \left(1-\frac{M}{r}\right)\left(\frac{\partial}{\partial r}\right)^{a}+\mathcal{O}\left(\frac{1}{r^{2}}\right)\left(\frac{\partial}{\partial r}\right)^{a}, \\
e_{\vartheta}^{a} \approx & \frac{1}{r}\left(\frac{\partial}{\partial \vartheta}\right)^{a}+\mathcal{O}\left(\frac{1}{r^{2}}\right) \frac{1}{r}\left(\frac{\partial}{\partial \vartheta}\right)^{a}, \\
e_{\varphi}^{a} \approx & \frac{1}{r \sin \vartheta}\left(\frac{\partial}{\partial \varphi}\right)^{a}+\mathcal{O}\left(\frac{1}{r^{2}}\right) \frac{1}{r \sin \vartheta}\left(\frac{\partial}{\partial \varphi}\right)^{a} .
\end{aligned}
$$

Omitting all corrections at or above the order of $M^{2} / r^{2}$, the metric takes the form

$$
\begin{aligned}
g_{a b} \approx & -\left(1-\frac{2 M}{r}\right) d t_{a} d t_{b}+\left(1+\frac{2 M}{r}\right) d r_{a} d r_{b} \\
& +r^{2} d \vartheta_{a} d \vartheta_{b}+r^{2} \sin ^{2} \vartheta d \varphi_{a} d \varphi_{b} .
\end{aligned}
$$

We write the polarization vector $f^{a}$ in terms of the ON tetrad,

$$
f^{a}=f^{\hat{r}} e_{r}^{a}+f^{\hat{\vartheta}} e_{\vartheta}^{a}+f^{\hat{\varphi}} e_{\varphi}^{a},
$$

where $f^{\hat{r}}, f^{\hat{\vartheta}}$, and $f^{\hat{\varphi}}$ must be finite. We have used the gauge freedom of $f^{a}$ to choose $f^{\hat{t}}=0$ at infinity. Then we have, as $r \rightarrow \infty$,

$$
\begin{gathered}
f_{t}=0, \quad f_{r} \approx\left(1+\frac{M}{r}\right) f^{\hat{r}}, \\
f_{\vartheta} \approx r f^{\hat{\vartheta}}, \quad f_{\varphi} \approx r \sin \vartheta f^{\hat{\varphi}} .
\end{gathered}
$$

Similarly, we can write the photon wavevector $k^{a}$ in terms of the ON tetrad. Expressed in $k_{\mu}$ (which must be finite), we have

$$
\begin{aligned}
k^{a} \approx & -\left(1+\frac{M}{r}\right) k_{t} e_{t}^{a}+\left(1-\frac{M}{r}\right) k_{r} e_{r}^{a} \\
& +\frac{1}{r} k_{\vartheta} e_{\vartheta}^{a}+\frac{1}{r \sin \varphi} k_{\varphi} e_{\varphi}^{a} \\
= & -k_{t} e_{t}^{a}+k_{r} e_{r}^{a}, \quad \text { as } r \rightarrow \infty .
\end{aligned}
$$

The condition $k_{a} k^{a}=0$ then implies that (note, $k_{r}>0$ )

$$
k_{r}=-k_{t}=E_{\infty}, \quad \text { as } r \rightarrow \infty,
$$

and Equation (B20) leads to

$$
f_{r} \approx-\frac{1}{r k_{r}}\left(k_{\vartheta} f^{\hat{\vartheta}}+\frac{1}{\sin \vartheta} k_{\varphi} f^{\hat{\varphi}}\right)=\mathcal{O}\left(\frac{1}{r}\right),
$$

where we have omitted terms of the order $r^{-2}$. 
Hence we have

$$
\begin{aligned}
\left(r^{2}+a^{2}\right)\left(k_{r} f_{t}-k_{t} f_{r}\right) \approx & \frac{r k_{t}}{k_{r}}\left(k_{\vartheta} f^{\hat{\vartheta}}+\frac{1}{\sin \vartheta} k_{\varphi} f^{\hat{\varphi}}\right) \\
& +\mathcal{O}(1), \\
a\left(k_{r} f_{\varphi}-k_{\varphi} f_{r}\right) \approx & a r \sin \vartheta k_{r} f^{\hat{\varphi}}+\mathcal{O}\left(\frac{1}{r}\right), \\
k_{\vartheta} f_{\varphi}-k_{\varphi} f_{\vartheta} \approx & r\left(\sin \vartheta k_{\vartheta} f^{\hat{\varphi}}-k_{\varphi} f^{\hat{\vartheta}}\right) \\
& +\mathcal{O}\left(\frac{1}{r^{2}}\right), \\
a \sin ^{2} \vartheta\left(k_{t} f_{\vartheta}-k_{\vartheta} f_{t}\right) \approx & \operatorname{ar} \sin ^{2} \vartheta k_{t} f^{\hat{\vartheta}}+\mathcal{O}\left(\frac{1}{r^{2}}\right) .
\end{aligned}
$$

Substituting these into Equation (B19), we obtain in the limit $r \rightarrow \infty$

$$
\begin{aligned}
K_{1}+i K_{2}= & \frac{k_{t}}{k_{r}}\left(k_{\vartheta} f^{\hat{\vartheta}}+\frac{1}{\sin \vartheta} k_{\varphi} f^{\hat{\varphi}}\right)+a \sin \vartheta k_{r} f^{\hat{\varphi}} \\
& +i\left(k_{\vartheta} f^{\hat{\varphi}}-\frac{k_{\varphi}}{\sin \vartheta} f^{\hat{\vartheta}}-a \sin \vartheta k_{t} f^{\hat{\vartheta}}\right) .
\end{aligned}
$$

Substituting $k_{r}=-k_{t}$ (Equation (B56)) into Equation (B62), we have

$$
K_{1}+i K_{2}=-\left(T f^{\hat{\vartheta}}+S f^{\hat{\varphi}}\right)-i\left(S f^{\hat{\vartheta}}-T f^{\hat{\varphi}}\right),
$$

where $S$ and $T$ are defined by Equations (B12) and (B13), respectively. From Equation (B63) we can solve for $f^{\hat{\vartheta}}$ and $f^{\hat{\varphi}}$,

$$
f^{\hat{\vartheta}}=-\frac{K_{2} S+K_{1} T}{S^{2}+T^{2}}, \quad f^{\hat{\varphi}}=-\frac{K_{1} S-K_{2} T}{S^{2}+T^{2}},
$$

where $S^{2}+T^{2}$ is given by Equation (B15) and is a constant. From Equations (B23) and (B64), we then have

$$
\left(f^{\hat{\vartheta}}\right)^{2}+\left(f^{\hat{\varphi}}\right)^{2}=\frac{K_{1}^{2}+K_{2}^{2}}{S^{2}+T^{2}}=1 .
$$

Since as $r \rightarrow \infty$ we have $f^{\hat{t}}=0$ and $f^{\hat{r}}=0$ (Equation (B57)); Equation (B22) is satisfied.

\section{B.3. Solution of the Polarization Vector at Infinity}

By Equations (B46), (B47), and (B64) we have

$$
\begin{aligned}
f_{\infty}^{\hat{\vartheta}}= & \frac{E_{\infty} \Gamma r}{g A_{0}^{1 / 2}\left(S^{2}+T^{2}\right)} \\
& \times\left\{f^{\theta}[S(X \sin \phi+Y \sin \theta)-T X \cos \theta \cos \phi]\right. \\
& \left.+f^{\phi}[T(X \sin \phi+Y \sin \theta)+S X \cos \theta \cos \phi]\right\}, \\
f_{\infty}^{\hat{\varphi}}= & \frac{E_{\infty} \Gamma r}{g A_{0}^{1 / 2}\left(S^{2}+T^{2}\right)} \\
& \times\left\{f^{\theta}[-T(X \sin \phi+Y \sin \theta)-S X \cos \theta \cos \phi]\right. \\
& \left.+f^{\phi}[S(X \sin \phi+Y \sin \theta)-T X \cos \theta \cos \phi]\right\},
\end{aligned}
$$

where $g \equiv E_{\infty} / E_{\text {loc }}$ is the photon redshift factor. Note that, on the right-hand sides of the above equations, $S$ and $T$ are evaluated at the remote observer (hence $\vartheta=i_{\text {disk }}$, the disk inclination angle), but all the other quantities are evaluated at the disk surface.

The solutions given in Equations (B66) and (B67) can be written in a matrix form

$$
\left(\begin{array}{c}
f_{\infty}^{\hat{\vartheta}} \\
f_{\infty}^{\hat{\varphi}}
\end{array}\right)=\left(\begin{array}{cc}
\cos \Phi_{\mathrm{GR}} & \sin \Phi_{\mathrm{GR}} \\
-\sin \Phi_{\mathrm{GR}} & \cos \Phi_{\mathrm{GR}}
\end{array}\right)\left(\begin{array}{c}
f^{\theta} \\
f_{\phi}
\end{array}\right),
$$

where

$$
\begin{aligned}
\cos \Phi_{\mathrm{GR}}=\xi_{x} \equiv & \frac{E_{\infty} \Gamma r}{g A_{0}^{1 / 2}\left(S^{2}+T^{2}\right)} \\
& \times[S(X \sin \phi+Y \sin \theta) \\
& -T X \cos \theta \cos \phi], \\
\sin \Phi_{\mathrm{GR}}=\xi_{y} \equiv & \frac{E_{\infty} \Gamma r}{g A_{0}^{1 / 2}\left(S^{2}+T^{2}\right)} \\
& \times[T(X \sin \phi+Y \sin \theta) \\
& +S X \cos \theta \cos \phi] .
\end{aligned}
$$

It can be checked that $\cos ^{2} \Phi_{\mathrm{GR}}+\sin ^{2} \Phi_{\mathrm{GR}}=1$ is fulfilled.

Define

$$
f_{\infty}^{\hat{\vartheta}}=\cos \psi_{\infty}, \quad f_{\infty}^{\hat{\varphi}}=\sin \psi_{\infty}
$$

and

$$
f^{\theta}=\cos \psi_{\mathrm{em}}, \quad f_{\phi}=\sin \psi_{\mathrm{em}} .
$$

Then Equation (B68) leads to

$$
\begin{aligned}
\cos \psi_{\infty} & =\cos \left(\psi_{\mathrm{em}}-\Phi_{\mathrm{GR}}\right), \\
\sin \psi_{\infty} & =\sin \left(\psi_{\mathrm{em}}-\Phi_{\mathrm{GR}}\right),
\end{aligned}
$$

whose solution is

$$
\psi_{\infty}=\psi_{\mathrm{em}}-\Phi_{\mathrm{GR}}+2 n \pi
$$

where $n=0, \pm 1, \pm 2, \cdots$. Hence, the $\Phi_{\mathrm{GR}}$ defined by Equations (B69) and (B70) represents the rotation of the polarization vector induced by the Kerr geometry and disk rotation.

We define $\Phi_{\mathrm{GR}}$ to be the primitive rotation angle, which satisfies $0 \leqslant \Phi_{\mathrm{GR}}<2 \pi$. Then by Equations (B69) and (B70) we have

$$
\Phi_{\mathrm{GR}}=\left\{\begin{array}{ll}
\arccos \xi_{x}, & \text { if } \xi_{y} \geqslant 0 \\
2 \pi-\arccos \xi_{x}, & \text { if } \xi_{y}<0
\end{array} .\right.
$$

We can check the nonrelativistic limit of this result in flat spacetime. For this purpose, let us take $a=0, \Omega=0$, $v_{\varphi}=0, \Gamma=1, L^{\dagger}=0, E^{\dagger}=1, \chi=1, g=1$, and $A_{0}=r^{4}$. Then we have $X=r^{2}$ and $Y=0$. Since the light ray is not bent, we have $\theta=\vartheta_{\text {obs }}=i_{\text {disk }}$. So, we have $L_{z}=E_{\infty} r \sin \theta \sin \phi, S=E_{\infty} r \sin \phi$, and $T=$ $\operatorname{sign}\left(k_{\vartheta_{\text {obs }}}\right) \sqrt{\mathcal{Q}-L_{z}^{2} \cot ^{2} \theta}=-E_{\infty} r \cos \theta \cos \phi$. Then $\xi_{x}=1$, $\xi_{y}=0$, and hence the polarization vector is not rotated. 
The calculation of $\Psi_{\mathrm{GR}}$ and $\psi_{\infty}$ is simplified by using dimensionless variables. We define dimensionless quantities by symbols with a tilde,

$$
\begin{array}{r}
X \equiv \tilde{X} r^{2}, \quad Y \equiv \tilde{Y} r^{2}, \quad S \equiv \tilde{S} r E_{\infty}, \\
T \equiv \tilde{T} r E_{\infty}, \quad A_{0} \equiv \tilde{A} r^{4} .
\end{array}
$$

Then, by Equations (B69) and (B70), we have

$$
\begin{aligned}
\xi_{x}= & \frac{\Gamma}{g \tilde{A}^{1 / 2}\left(\tilde{S}^{2}+\tilde{T}^{2}\right)} \\
& \times[\tilde{S}(\tilde{X} \sin \phi+\tilde{Y} \sin \theta)-\tilde{T} \tilde{X} \cos \theta \cos \phi], \\
\xi_{y}= & \frac{\Gamma}{g \tilde{A}^{1 / 2}\left(\tilde{S}^{2}+\tilde{T}^{2}\right)} \\
& \times[\tilde{T}(\tilde{X} \sin \phi+\tilde{Y} \sin \theta)+\tilde{S} \tilde{X} \cos \theta \cos \phi],
\end{aligned}
$$

where

$$
\begin{gathered}
\tilde{X}=1+\frac{a^{2}}{r^{2}}-\frac{a}{r}\left(\frac{\Delta}{r^{2}}\right)^{1 / 2} v_{\varphi}, \\
\tilde{Y}=\left(1+\frac{a^{2}}{r^{2}}\right) v_{\varphi}-\frac{a}{r}\left(\frac{\Delta}{r^{2}}\right)^{1 / 2},
\end{gathered}
$$

and

$$
\begin{aligned}
& \tilde{S}=\frac{\lambda}{r \sin \vartheta_{\mathrm{obs}}}-\frac{a}{r} \sin \vartheta_{\mathrm{obs}}, \\
& \tilde{T}=\operatorname{sign}\left(k_{\vartheta_{\mathrm{obs}}}\right) \frac{1}{r} \sqrt{Q+a^{2} \cos ^{2} \vartheta_{\mathrm{obs}}-\lambda^{2} \cot ^{2} \vartheta_{\mathrm{obs}}},
\end{aligned}
$$

where $\lambda \equiv L_{z} / E_{\infty}$, and $Q \equiv \mathcal{Q} / E_{\infty}^{2}$ are independent of $E_{\infty}$ (Li et al. 2005).

\section{REFERENCES}

Afshordi, N., \& Paczyński, B. 2003, ApJ, 592, 354

Agol, E., Blaes, O., \& Ionescu-Zanetti, C. 1998, MNRAS, 293, 1

Agol, E., \& Krolik, J. H. 2000, ApJ, 528, 161

Alfvén, H., \& Herlofson, N. 1950, Phys. Rev., 78, 616

Antonucci, R. R. J., \& Miller, J. S. 1985, ApJ, 297, 621

Bardeen, J. M., \& Petterson, J. A. 1975, ApJ, 195, L65

Bardeen, J. M., Press, W. H., \& Teukolsky, S. A. 1972, ApJ, 178, 347

Beckwith, K., Hawley, J. F., \& Krolik, J. H. 2008, ApJ, 678, 1180

Blandford, R., Agol, E., Broderick, A., Heyl, J., Koopmans, L., \& Lee,

H.-W. 2002, in Proc. XII Canary Islands Winter School of Astrophysics, Astrophysical Spectropolarimetry, ed. J. Trujillo-Bueno, F. Moreno Insertis, \& F. Sánchez (Cambridge: Cambridge Univ. Press), 177

Brenneman, L. W., \& Reynolds, C. S. 2006, ApJ, 652, 1028

Carter, B. 1968, Phys. Rev., 174, 1559

Chandrasekhar, S. 1960, Radiative Transfer (New York: Dover)

Chandrasekhar, S. 1983, The Mathematical Theory of Black Holes (New York: Oxford Univ. Press)

Charles, P. A., \& Coe, M. J. 2006, in Compact Stellar X-Ray Sources, ed. W. H. G. Lewin \& M. van der Klis (Cambridge: Cambridge Univ. Press), 215

Chen, K., \& Eardley, D. M. 1991, ApJ, 382, 125

Connors, P. A., Piran, T., \& Stark, R. F. 1980, ApJ, 235, 224

Connors, P. A., \& Stark, R. F. 1977, Nature, 269, 128

Costa, E., Soffitta, P., Bellazzini, R., Brez, A., Lumb, N., \& Spandre, G. 2001, Nature, 411, 662

Davis, S. W., Blaes, O. M., Hubeny, I., \& Turner, N. J. 2005, ApJ, 621, 372

Davis, S. W., Done, C., \& Blaes, O. M. 2006, ApJ, 647, 525

Davis, S. W., \& Hubeny, I. 2006, ApJS, 164, 530

Dovčiak, M., Karas, V., \& Matt, G. 2004, MNRAS, 355, 1005

Dovčiak, M., Muleri, F., Goosmann, R. W., Karas, V., \& Matt, G. 2008, MNRAS, 391,22
Ebisawa, K., et al. 1994, PASJ, 46, 375

Fabian, A. C., Iwasawa, K., Reynolds, C. S., \& Young, A. J. 2000, PASP, 112 1145

Fragile, P. C., \& Anninos, P. 2005, ApJ, 623, 347

Fragile, P. C., Blaes, O. M., Anninos, P., \& Salmonson, J. D. 2007, ApJ, 668, 417

Frank, J., King, A., \& Raine, D. 2002, Accretion Power in Astrophysics, (Cambridge: Cambridge Univ. Press)

Fryer, C. L., \& Kalogera, V. 2001, ApJ, 554, 548

Fryer, C. L., \& Young, P. A. 2007, ApJ, 659, 1438

Gierliński, M., Zdziarski, A. A., Poutanen, J., Coppi, Paolo S., Ebisawa, K., \& Johnson, W. N. 1999, MNRAS, 309, 496

Grove, J. E., Johnson, W. N., Kroeger, R. A., McNaron-Brown, K., Skibo, J. G., \& Phlips, B. F. 1998, ApJ, 500, 899

Hjellming, R. M., \& Rupen, M. P. 1995, Nature, 375, 464

Hjellming, R. M., et al. 2000, ApJ, 544, 977

Hubeny, I. 1990, ApJ, 351, 632

King, A. R., Lubow, S. H., Ogilvie, G. I., \& Pringle, J. E. 2005, MNRAS, 363 , 49

Krolik, J. H. 1999, ApJ, 515, L73

Krolik, J. H., \& Hawley, J. F. 2002, ApJ, 573, 754

Krolik, J. H., Hawley, J. F., \& Hirose, S. 2005, ApJ, 622, 1008

Laor, A., Netzer, H., \& Piran, T. 1990, MNRAS, 242, 560

Li, L.-X. 2002, Phys. Rev. D, 67, 044007

Li, L.-X., Zimmerman, E. R., Narayan, R., \& McClintock, J. 2005, ApJS, 157, 335

Lightman, A. P., \& Shapiro, S. L. 1975, ApJ, 198, L73

Liu, J., McClintock, J. E., Narayan, R., Davis, S. W., \& Orosz, J. A. 2008, ApJ, 679, L37

Lodato, G., \& Pringle, J. E. 2006, MNRAS, 368, 1196

Maccarone, T. J. 2002, MNRAS, 336, 1371

Martin, R. G., Pringle, J. E., \& Tout, C. A. 2007, MNRAS, 381, 1617

Matilsky, T., et al. 1976, ApJ, 210, L127

McClintock, J. E., Narayan, R., \& Shafee, R. 2008, in Black Holes, ed. M. Livio \& A. Koekemoer (Cambridge Univ. Press), in press (arXiv:0707.4492)

McClintock, J. E., \& Remillard, R. A. 2006, in Compact Stellar X-Ray Sources, ed. W. H. G. Lewin \& M. van der Klis (Cambridge: Cambridge Univ. Press), 157

McClintock, J. E., Shafee, R., Narayan, R., Remillard, R. A., Davis, S. W., \& Li, L.-X. 2006, ApJ, 652, 518

Mészáros, P., Novick, R., Chanan, G. A., Weisskopf, M. C., \& Szentgyörgyi, A. 1988, ApJ, 324, 1056

Miller, J. M. 2007, ARA\&A, 45, 441

Miller, J. M., et al. 2008, ApJ, 679, L113

Mirabel, I. F., \& Rodríguez, L. F. 1999, ARA\&A, 37, 409

Misner, C. W., Thorne, K. S., \& Wheeler, J. A. 1973, Gravitation (New York: Freeman)

Narayan, R., \& McClintock, J. E. 2005, ApJ, 623, 1017

Narayan, R., McClintock, J. E., \& Shafee, R. 2008, in Astrophysics of Compact Objects, ed. Y. F. Yuan, X. D. Li, \& D. Lai (New York: AIP), 265

Noble, S. C., Krolik, J. H., \& Hawley, J. F. 2008, ApJ, submitted (arXiv:0808.3140)

Novick, R., Weisskopf, M. C., Berthelsdorf, R., Linke, R., \& Wolff, R. S. 1972, ApJ, 174, L1

Novikov, I. D., \& Thorne, K. S. 1973, in Black Holes, ed. C. DeWitt \& B. S. DeWitt (New York: Gordon and Breach), 343

Orosz, J. A. 2003, in Massive Star Odyssey: From Main Sequence to Supernova, ed. K. van der Hucht, A. Herrero, \& C. Esteban (San Francisco, CA: ASP), 365

Orosz, J. A., et al. 2001, ApJ, 555, 489

Orosz, J. A., et al. 2007, Nature, 449, 872

Paczyński, B. 2000, arXiv:astro-ph/0004129

Page, D. N., \& Thorne, K. S. 1974, ApJ, 191, 499

Popham, R., \& Narayan, R. 1995, ApJ, 442, 337

Rees, M. J. 1975, MNRAS, 171, 457

Remillard, R. A., \& McClintock, J. E. 2006, ARA\&A, 44, 49

Reynolds, C. S., \& Fabian, A. C. 2008, ApJ, 675, 1048

Ross, R. R., Fabian, A. C., \& Young, A. J. 1999, MNRAS, 306, 461

Schnittman, J. D. 2005, ApJ, 621, 940

Shafee, R., McClintock, J. E., Narayan, R., Davis, S. W., Li, L.-X., \& Remillard, R. A. 2006, ApJ, 636, 113

Shafee, R., Mc Kinney, J. C., Narayan, R., Tchekhovskoy, A., Gammie, C. F., \& McClintock, J. E. 2008a, ApJ, 687, L25

Shafee, R., Narayan, R., \& McClintock, J. E. 2008b, ApJ, 676, 549

Shakura, N. I., \& Sunyaev, R. A. 1973, A\&A, 24, 337

Shimura, T., \& Takahara, F. 1995, ApJ, 445, 780

Silverman, J. M., \& Filippenko, A. V. 2008, ApJ, 678, L17 
Stark, R. F., \& Connors, P. A. 1977, Nature, 266, 429

Swank, J. H., et al. 2004, Presentation at the X-Ray Polarimetry Workshop held at SLAC (http://heasarc.gsfc.nasa.gov/docs/heasarc/polar/polar.html)

Török, G., Abramowicz, M. A., Kluzńiak, W., \& Stuchlík, Z. 2005, A\&A, 436, 1

Tsunemi, H., Kitamoto, S., Okamura, S., \& Roussel-Dupré, D. 1989, ApJ, 336, L81

van Dam, M. A., et al. 2006, PASP, 118, 310

Vernet, J., et al. 2007, ESO Messenger No. 130 (2007 December), 5
Wagoner, R. V., Silbergleit, A. S., \& Ortega-Rodríguez, M. 2001, ApJ, 559, 25

Wald, R. 1984, General Relativity (Chicago: Univ. Chicago Press)

Walker, M., \& Penrose, R. 1970, Commun. Math. Phys., 18, 265

Watson, M. G., Ricketts, M. J., \& Griffiths, R. E. 1978, ApJ, 221, L69

Weisskopf, M. C., Silver, E. H., Kestenbaum, H. L., Long, K. S., \& Novick, R. 1978, ApJ, 220, L117

Wilms, J., Nowak, M. A., Pottschmidt, K., Pooley, G. G., \& Fritz, S. 2006, A\&A, 447,245

Zahn, J.-P. 1977, ApJ, 57, 383 